Canad. J. Math. Vol. 71 (3), 2019 pp. 659-681

http://dx.doi.org/10.4153/CJM-2018-002-2

(C) Canadian Mathematical Society 2018

\title{
Freeness and The Partial Transposes of Wishart Random Matrices
}

\author{
James A. Mingo and Mihai Popa
}

Abstract. We show that the partial transposes of complex Wishart random matrices are asymptotically free. We also investigate regimes where the number of blocks is fixed but the size of the blocks increases. This gives an example where the partial transpose produces freeness at the operator level. Finally, we investigate the case of real Wishart matrices.

\section{Introduction}

Suppose we have a matrix $A$ in $M_{d_{1}}(\mathbb{C}) \otimes M_{d_{2}}(\mathbb{C})$. We can write this as a block matrix.

$$
A=\left(\begin{array}{c|c|c}
A(1,1) & \cdots & A\left(1, d_{1}\right) \\
\hline \vdots & & \vdots \\
\hline A\left(d_{1}, 1\right) & \cdots & A\left(d_{1}, d_{1}\right)
\end{array}\right) .
$$

with each $A(i, j) \in M_{d_{2}}(\mathbb{C})$. We can form two partial transposes of this matrix.

$$
A^{\mathrm{T}}=\left(\begin{array}{c|c|c}
A(1,1) & \cdots & A\left(d_{1}, 1\right) \\
\hline \vdots & & \vdots \\
\hline A\left(1, d_{1}\right) & \cdots & A\left(d_{1}, d_{1}\right) .
\end{array}\right), \quad A^{\Gamma}=\left(\begin{array}{cc|c|c}
A(1,1)^{\mathrm{T}} & \cdots & A\left(1, d_{1}\right)^{\mathrm{T}} \\
\hline \vdots & & \vdots \\
\hline A\left(d_{1}, 1\right)^{\mathrm{T}} & \cdots & A\left(d_{1}, d_{1}\right)^{\mathrm{T}} .
\end{array}\right) .
$$

In quantum information theory the partial transpose has been used as an entanglement detector. Suppose that $A$ is a positive matrix in $M_{d_{1}}(\mathbb{C}) \otimes M_{d_{2}}(\mathbb{C})$. Recall that $A$ is entangled if we cannot find positive matrices $B_{1}, \ldots, B_{k} \in M_{d_{1}}(\mathbb{C})$ and $C_{1}, \ldots, C_{k} \in M_{d_{2}}(\mathbb{C})$ such that $A=\sum_{i=1}^{k} A_{i} \otimes B_{i}$. If a positive matrix fails to have a positive partial transpose, then the matrix must be entangled. It was shown by Aubrun [2] that in a particular regime of the Wishart distribution, the partial transpose of a positive matrix is typically entangled. He also showed that the limiting distribution of a partially transposed Wishart matrix is semi-circular. This was quite unexpected. We revisit his theorem and show that the conclusion can be explained by the requirement that the non-crossing partitions that survive in the limit have to remain non-crossing when the order of elements is reversed.

Received by the editors August 27, 2017; revised January 7, 2018.

Published electronically April 12, 2018.

Research of both authors was supported by a Discovery Grant from the Natural Sciences and Engineering Research Council of Canada. Research of author M.P. was also supported by the Simons Foundation, grant No. 360242.

AMS subject classification: 15B52, 46L54, 60B20.

Keywords: free probability, random matrix, partial transpose, quantum information theory. 
In this paper we show that in addition to transforming a Marchenko-Pastur into a semi-circular distribution, the partial transpose also produces freeness in two different regimes. The first is when both $d_{1}$ and $d_{2}$ tend to infinity; we show that when $W$ is a Wishart matrix, $W, W^{\mathrm{T}}, W^{\mathrm{\Gamma}}$, and $W^{\mathrm{T}}$ are asymptotically free in the complex case and that $W=W^{\mathrm{T}}$ and $W^{\mathrm{\Gamma}}=W^{\mathrm{T}}$ are asymptotically free in the real case.

The second regime is the one considered by Banica and Nechita [3] where we fix $d_{1}$ and let $d_{2}$ tend to $\infty$. In this case we show that $W$ and $W^{\Gamma}$ are asymptotically free. As $\left(W^{\mathrm{T}}\right)^{\mathrm{T}}=W^{\mathrm{T}}$, one has that $W^{\mathrm{T}}$ and $W^{\mathrm{T}}$ have the same limit distributions. Banica and Nechita showed that the limit distribution of $d_{1} W^{\mathrm{T}}$ could be written as the free difference of two Marchenko-Pastur distributions. We can show that in the same regime and when $d_{1}=2$, one can write the limit distribution of $d_{1} W^{\mathrm{T}}$ as the sum of two free operators $X_{1}$ and $X_{2}$ coming from the diagonal and off-diagonal parts of $d_{1} W^{\mathrm{T}}$. More precisely, if we write a Marchenko-Pastur random variable $w$ as a block matrix

$$
w=\frac{1}{2}\left(\begin{array}{ll}
w_{11} & w_{12} \\
w_{21} & w_{22}
\end{array}\right), \quad X_{1}=\left(\begin{array}{cc}
w_{11} & 0 \\
0 & w_{22}
\end{array}\right), \quad X_{1}=\left(\begin{array}{cc}
0 & w_{21} \\
w_{12} & 0
\end{array}\right)
$$

then $d_{1} w^{\mathrm{T}}=X_{1}+X_{2}$ and $X_{1}$ and $X_{2}$ are free. Moreover, $X_{1}$ is a Marchenko-Pastur random variable with the same distribution as $2 w$, and $X_{2}$ is an even operator whose even cumulants are the same as those of $X_{1}$. So, by writing $d_{1} w^{\mathrm{T}}$ as a sum as opposed to a difference, we get a natural representation for the two operators.

The connection of the transpose to freeness goes back to the work of Emily Redelmeier on real second order freeness [13]. She showed that the fluctuation moments of real Gaussian and Wishart matrices require the transpose to be taken into account. Later in [9] the authors showed that the transpose can also appear at the first order level. Namely, that for many complex ensembles a random matrix could be asymptotically free from its transpose. Before this, the known examples of asymptotic freeness required independence of the entries; see [10, Ch. 4] and [11, Lect. 23, 24] for examples.

Our main tool here is the explicit evaluation of the mixed moments of the four matrices $W, W^{\mathrm{T}}, W^{\mathrm{T}}$, and $W^{\mathrm{T}}$ and then to show that mixed cumulants must vanish, thus demonstrating freeness. In order to achieve this we use the technique of doubling of indices, which already appeared in the work of Redelmeier [12,13].

Besides the transpose one can consider the action of other positive linear maps on the blocks of matrices and the effect on the limit eigenvalue distribution. This was considered in considerable generality in the recent paper of Arizmendi, Nechita, and Vargas [1]. A third regime was considered by Fukuda and Śniady in [6] and a connection to meander polynomials was found.

The outline of the paper is as follows. In section 2 we establish the notation needed for our calculations. The main method for computing mixed moments is to expand the expression as a sum over the symmetric group. This part is presented in Theorem 3.7. In Section 4 we determine which permutations contribute to the limit. The main result in this section is Proposition 4.7. In Section 5 we consider the limit distributions of our partially transposed operators in the two regimes. In particular, we will show that the operators $X_{1}$ and $X_{2}$ mentioned above are free. In Section 7 we 
present our main results, the asymptotic freeness of our partially transposed Wishart matrices. In Section 8 we consider the situation for real Wishart matrices.

\section{Notation and Statement of Results}

Suppose $G_{1}, \ldots, G_{d_{1}}$ are $d_{2} \times p$ random matrices where $G_{i}=\left(g_{j k}^{(i)}\right)_{j k}$ and $g_{j k}^{(i)}$ are complex Gaussian random variables with mean 0 and (complex) variance 1, i.e., $\mathrm{E}\left(\left|g_{j k}^{(i)}\right|^{2}\right)=1$. Moreover, suppose that the random variables $\left.g_{j k}^{(i)}\right\}_{i, j, k}$ are independent. Then

$$
W=\frac{1}{d_{1} d_{2}}\left(\frac{\frac{G_{1}}{\vdots}}{G_{d_{1}}}\right)\left(G_{1}^{*}|\cdots| G_{d_{1}}^{*}\right)=\frac{1}{d_{1} d_{2}}\left(G_{i} G_{j}^{*}\right)_{i j}
$$

is a $d_{1} d_{2} \times d_{1} d_{2}$ complex Wishart matrix. We write $W=d_{1}^{-1}(W(i, j))_{i j}$ as a $d_{1} \times d_{1}$ block matrix with each entry the $d_{2} \times d_{2}$ matrix $d_{2}^{-1} G_{i} G_{j}^{*}$. From this we get four matrices $\left\{W, W^{\mathrm{T}}, W^{\mathrm{\Gamma}}, W^{\mathrm{T}}\right\}$ defined as follows:

- $W^{\mathrm{T}}=\frac{1}{d_{1}}\left(W(j, i)^{\mathrm{T}}\right)_{i j}$ is the "full" transpose

- $W^{\mathrm{T}}=\frac{1}{d_{1}}(W(j, i))_{i j}$ is the "left" partial transpose

- $W^{\Gamma}=\frac{1}{d_{1}}\left(W(i, j)^{\mathrm{T}}\right)_{i j}$ is the "right" partial transpose

Note that the $X \mapsto X^{\Gamma}$ notation conceals the dependence on $d_{1}$ and $d_{2}$. Thus, as the size of the matrix grows, these operators might be expected to behave differently, depending on the way $d_{1}$ and $d_{2}$ grow.

If the random variables $\left\{g_{j k}^{(i)}\right\}_{i, j, k}$ are real Gaussian random variables with mean 0 and variance 1 , then $W$ is a real Wishart matrix. For many eigenvalue results there is no distinction between the real and complex case. In [9] we showed that when it comes to freeness there is a difference, in particular with respect to the behaviour of the transpose. In this paper we show that with the partial transpose we continue to see a difference between the real and complex cases.

If we assume that $d_{1}, d_{2} \rightarrow \infty$ and that $\frac{p}{d_{1} d_{2}} \rightarrow c, 0<c<\infty$, then the eigenvalue distributions of $W$ and $W^{\mathrm{T}}$ converge to Marchenko-Pastur with parameter $c$. This is the distribution on $\mathbb{R}^{+}$that has density $\sqrt{(b-t)(t-a)} / 2 \pi t$ on $[a, b]$ and an atom of weight $(1-c)$ at 0 if $c<1$; we set $b=(1+\sqrt{c})^{2}$ and $a=(1-\sqrt{c})^{2}$.

Note that we are using what one might call the free probabilist's Marchenko-Pastur law. In our normalization, all the cumulants are equal to $c$. For the relation between the two see [10, Ch. 2 Remark 12]. With this normalization we can restate Aubrun's theorem.

Suppose $\lim _{d_{1}, d_{2} \rightarrow \infty} \frac{p}{d_{1} d_{2}}=c$, then the eigenvalue distributions of $W^{\mathrm{T}}$ and $W^{\Gamma}$ converge to a shifted semi-circular operator with mean $c$ and variance $c$.

Our main results are the following theorems.

Theorem 2.1 Suppose $\lim _{d_{1}, d_{2} \rightarrow \infty} \frac{p}{d_{1} d_{2}}=c$; then the family $\left\{W, W^{\mathrm{T}}, W^{\mathrm{\Gamma}}, W^{\mathrm{T}}\right\}$ is asymptotically free in the complex case, and the family $\left\{W, W^{\Gamma}\right\}$ is asymptotically free in the real case. 
Theorem 2.2 Suppose $d_{1}$ is fixed and $\lim _{d_{2} \rightarrow \infty} \frac{p}{d_{1} d_{2}}=c$; then the family $\left\{W, W^{\mathrm{\Gamma}}\right\}$ is asymptotically free in the complex case.

In $M_{2}(\mathbb{C})$, let $\left\{E_{i j}\right\}_{i j=1}^{2}$ be the standard matrix units. For convenience of notation we write $E_{i j}$ for $E_{i j} \otimes I_{d_{2}} \in M_{2}(\mathbb{C}) \otimes M_{d_{2}}(\mathbb{C})$.

Theorem 2.3 Suppose $d_{1}=2, p, d_{2} \rightarrow \infty$, and $\lim _{d_{2} \rightarrow \infty} \frac{p}{d_{1} d_{2}}=c$. Then $\left\{W, W^{\mathrm{T}}, E_{i j}\right\}_{i,=1}^{2}$ has a limit joint distribution, $\left\{w, w^{\mathrm{T}}, e_{i j}\right\}_{i, j=1}^{2}$, in a non-commutative *-probability space $(\mathcal{A}, \varphi)$. Relative to the matrix units $\left\{e_{i j}\right\}_{i, j=1}^{2}$, we write

$$
w=\frac{1}{2}\left(\begin{array}{ll}
w_{11} & w_{12} \\
w_{21} & w_{22}
\end{array}\right) \quad \text { and } \quad w^{\mathrm{T}}=\frac{1}{2}\left(\begin{array}{ll}
w_{11} & w_{21} \\
w_{12} & w_{22}
\end{array}\right) .
$$

Then $X_{1}=\left(\begin{array}{cc}w_{11} & 0 \\ 0 & w_{22}\end{array}\right)$ and $X_{2}=\left(\begin{array}{cc}0 & w_{21} \\ w_{12} & 0\end{array}\right)$ are free, $X_{1}$ has a Marchenko-Pastur distribution with parameter $2 c$, and $X_{2}$ is an even operator with all even cumulants equal to $2 c$.

\section{A General Formula for Mixed Moments}

Let $A_{1}, \ldots, A_{n}$ be $N \times N$ matrices. Then

$$
\operatorname{Tr}\left(A_{1} \cdots A_{n}\right)=\sum_{i_{ \pm 1}, \ldots, i_{ \pm n}=1}^{N} a_{i_{1}, i_{-1}}^{(1)} a_{i_{2}, i_{-2}}^{(2)} \cdots a_{i_{n} i_{-n}}^{(n)}
$$

where the sum runs over all $i:[ \pm n] \rightarrow[N]$ such that

$$
i(-1)=i(2), \quad i(-2)=i(3), \ldots, i(-n)=i(1) .
$$

Remark 3.1 We wish to use the symmetric group $S_{n}$ of permutations on $[n]=$ $\{1,2,3, \ldots, n\}$ to keep track of the partial transposes. So we introduce the following notation. Given a permutation $\sigma$ in $S_{n}$, we extend $\sigma$ to be a permutation on $[ \pm n]=\{1,-1,2,-2,3,-3, \ldots, n,-n\}$ by setting $\sigma(-k)=-k$ for $k>0$. We let $\delta$ be the permutation of $[ \pm n]$ given by $\delta(k)=-k$ for all $k \in[ \pm n]$ and $\gamma \in S_{n}$ be the permutation with one cycle: $\gamma=(1,2,3, \ldots, n)$. With our conventions we have $\gamma \delta \gamma^{-1}=(1,-n)(2,-1)(3,-2) \cdots(n,-(n-1))$. The condition in (3.1) now becomes $i=i \circ \gamma \delta \gamma^{-1}$.

To show that the family $\left\{W, W^{\mathrm{T}}, W^{\mathrm{T}}, W^{\mathrm{T}}\right\}$ is asymptotically free, we have to compute the expectation of the trace of arbitrary words in $\left\{W, W^{\mathrm{T}}, W^{\mathrm{T}}, W^{\mathrm{T}}\right\}$. For this we use the following notation.

Let $(\epsilon, \eta) \in\{-1,1\}^{2}=\mathbb{Z}_{2}^{2}$. We set

$$
W^{(\epsilon, \eta)}= \begin{cases}W & \text { if }(\epsilon, \eta)=(1,1), \\ W^{\mathrm{T}} & \text { if }(\epsilon, \eta)=(-1,1), \\ W^{\mathrm{T}} & \text { if }(\epsilon, \eta)=(1,-1), \\ W^{\mathrm{T}} & \text { if }(\epsilon, \eta)=(-1,-1) .\end{cases}
$$


Let $\left(\epsilon_{1}, \eta_{1}\right), \ldots,\left(\epsilon_{n}, \eta_{n}\right) \in \mathbb{Z}_{2}^{n}$, then an arbitrary word in $\left\{W, W^{\mathrm{T}}, W^{\mathrm{\Gamma}}, W^{\mathrm{T}}\right\}$ is $W^{\left(\epsilon_{1}, \eta_{1}\right)} \ldots W^{\left(\epsilon_{n}, \eta_{n}\right)}$ and we seek to write $\lim _{d_{1}, d_{2} \rightarrow \infty} \mathrm{E}\left(\operatorname{tr}\left(W^{\left(\epsilon_{1}, \eta_{1}\right)} \ldots W^{\left(\epsilon_{n}, \eta_{n}\right)}\right)\right)$ as a sum of free cumulants.

To achieve this we need to introduce still more notation. We shall suppose that $n$, the length of the word, is fixed for the moment. Now given $\left(\epsilon_{1}, \epsilon_{2}, \ldots, \epsilon_{n}\right)$ we denote by $\epsilon$ the permutation of $[ \pm n]$ given by $\epsilon(k)=\epsilon_{|k|} k$; here $k \epsilon[ \pm n]$, but $|k|>0$, so $\epsilon_{|k|}$ means the $k^{\text {th }}$ element of our vector $\left(\epsilon_{1}, \ldots, \epsilon_{n}\right)$. Similarly given $\left(\eta_{1}, \ldots, \eta_{n}\right)$ we get the permutation $\eta$ of $[ \pm n]$. Note that $\delta, \epsilon$ and $\eta$ all commute with each other.

We shall think of $W, W^{\mathrm{T}}, W^{\mathrm{T}}$, and $W^{\mathrm{T}}$ as random elements of $M_{d_{1}}(\mathbb{C}) \otimes M_{d_{2}}(\mathbb{C})$. On this algebra we have a trace $\operatorname{Tr} \otimes \operatorname{Tr}$; we also have the normalized trace $\operatorname{tr} \otimes \operatorname{tr}=$ $\frac{1}{d_{1} d_{2}} \operatorname{Tr} \otimes \operatorname{Tr}$.

Lemma 3.2 With the notations above, we have that

$$
\begin{aligned}
& \mathrm{E}\left(\operatorname{Tr} \otimes \operatorname{Tr}\left(W^{\left(\epsilon_{1}, \eta_{1}\right)} \ldots W^{\left(\epsilon_{n}, \eta_{n}\right)}\right)\right)= \\
& \quad\left(d_{1} d_{2}\right)^{-n} \sum_{j_{ \pm 1}, \ldots, j_{ \pm n}} \sum_{s_{ \pm 1}, \ldots, s_{ \pm n}} \sum_{t_{1}, \ldots, t_{n}} \mathrm{E}\left(g_{s_{1} t_{1}}^{\left(j_{1}\right)} \cdots g_{s_{n} t_{n}}^{\left(j_{n}\right)} \overline{g_{s_{-1} t_{1}}^{\left(j_{-1}\right)}} \cdots \overline{g_{s_{-n} t_{n}}^{\left(j_{-n}\right)}}\right),
\end{aligned}
$$

where the summation is subject to the conditions that $j=j \circ \epsilon \gamma \delta \gamma^{-1} \epsilon, s=s \circ \eta \gamma \delta \gamma^{-1} \eta$.

Proof We have

$$
\begin{aligned}
& \operatorname{Tr} \otimes \operatorname{Tr}\left(W^{\left(\epsilon_{1}, \eta_{1}\right)} \ldots W^{\left(\epsilon_{n}, \eta_{n}\right)}\right) \\
& =d_{1}^{-n} \sum_{i_{1}, \ldots, i_{n}=1}^{d_{1}} \operatorname{Tr}\left(\left(W^{\left(\epsilon_{1}, \eta_{1}\right)}\right)_{i_{1} i_{2}} \cdots\left(W^{\left(\epsilon_{n}, \eta_{n}\right)}\right)_{i_{n} i_{1}}\right) \\
& =d_{1}^{-n} \sum_{\substack{i_{ \pm 1}, \ldots, i_{ \pm n}=1 \\
i=i \circ \gamma \delta \gamma^{-1}}}^{d_{1}} \operatorname{Tr}\left(\left(W^{\left(\epsilon_{1}, \eta_{1}\right)}\right)_{i_{1} i_{-1}} \cdots\left(W^{\left(\epsilon_{n}, \eta_{n}\right)}\right)_{i_{n} i_{-n}}\right) \\
& =d_{1}^{-n} \sum_{\substack{j_{ \pm 1}, \ldots, j_{ \pm n}=1 \\
j=j \circ \epsilon \gamma \delta \gamma^{-1} \epsilon}}^{d_{1}} \operatorname{Tr}\left(W\left(j_{1}, j_{-1}\right)^{\left(\eta_{1}\right)} \cdots W\left(j_{n}, j_{-n}\right)^{\left(\eta_{n}\right)}\right) .
\end{aligned}
$$

To achieve the last step we let $j=i \circ \epsilon$. Also we have adopted the convention that for $A$ a matrix in $M_{d_{2}}(\mathbb{C})$ we let $A^{(1)}=A$ and $A^{(-1)}=A^{\mathrm{T}}$.

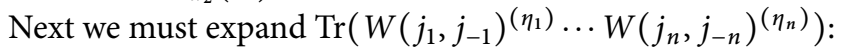

$$
\begin{aligned}
\operatorname{Tr}( & \left.W\left(j_{1}, j_{-1}\right)^{\left(\eta_{1}\right)} \cdots W\left(j_{n}, j_{-n}\right)^{\left(\eta_{n}\right)}\right) \\
& =\sum_{\substack{r_{ \pm 1}, \ldots, r_{ \pm n}=1 \\
r=r \circ \gamma \delta \gamma^{-1}}}^{d_{2}}\left(W\left(j_{1}, j_{-1}\right)^{\left(\eta_{1}\right)}\right)_{r_{1} r_{-1}} \cdots\left(W\left(j_{n}, j_{-n}\right)^{\left(\eta_{n}\right)}\right)_{r_{n} r_{-n}} \\
& =\sum_{\substack{s_{ \pm 1}, \ldots, s_{ \pm n}=1 \\
s=s \circ \eta \gamma \delta \gamma^{-1} \eta}}^{d_{2}}\left(W\left(j_{1}, j_{-1}\right)\right)_{s_{1} s_{-1}} \cdots\left(W\left(j_{n}, j_{-n}\right)\right)_{s_{n} s_{-n}}
\end{aligned}
$$




$$
\begin{aligned}
& =d_{2}^{-n} \sum_{s_{ \pm 1}, \ldots, s_{ \pm n}}\left(G_{j_{1}} G_{j_{-1}}^{*}\right)_{s_{1} s_{-1}} \cdots\left(G_{j_{n}} G_{j_{-n}}^{*}\right)_{s_{n} s_{-n}} \\
& =d_{2}^{-n} \sum_{s_{ \pm 1}, \ldots, s_{ \pm n}} \sum_{t_{1}, \ldots, t_{n}=1}^{p} g_{s_{1} t_{1}}^{\left(j_{1}\right)} \overline{g_{s_{-1} t_{1}}^{\left(j_{-1}\right)}} \cdots g_{s_{n} t_{n}}^{\left(j_{n}\right)} \overline{g_{s_{-n} t_{n}}^{\left(j_{-n}\right)}} .
\end{aligned}
$$

Hence the conclusion follows.

Next we need to compute $\mathrm{E}\left(g_{s_{1} t_{1}}^{\left(j_{1}\right)} \cdots g_{s_{n} t_{n}}^{\left(j_{n}\right)} \overline{g_{s_{-1} t_{1}}^{\left(j_{-1}\right)}} \cdots \overline{g_{s_{-n} t_{n}}^{\left(j_{-n}\right)}}\right)$. We shall use the complex form of Wick's rule that says that if $g_{1}, \ldots, g_{m}$ are independent $\mathcal{N}(0,1)$ random variables and $\alpha_{1}, \ldots, \alpha_{n}, \beta_{1}, \ldots, \beta_{n} \in[m]$, then $\mathrm{E}\left(g_{\alpha(1)} \cdots g_{\alpha(n)} \overline{g_{\beta(1)}} \cdots \overline{g_{\beta(n)}}\right)$ is the number of permutations $\sigma \in S_{n}$ such that for all $k \in[n]$ we have $\beta(k)=\alpha(\sigma(k))$; see Janson [7, p. 13].

Thus, we let $g_{\alpha(k)}=g_{s_{k} t_{k}}^{\left(j_{k}\right)}$ and $g_{\beta(k)}=g_{s_{-k} t_{k}}^{\left(j_{-k}\right)}$. So if $\sigma \in S_{n}$ and $\beta(k)=\alpha(\sigma(k))$, we have

$$
\begin{aligned}
s(-k) & =s(\sigma(k)) & & \text { for } k>0, \\
j(-k) & =j(\sigma(k)) & & \text { for } k>0, \\
t & =t(\sigma(k)) & & \text { for } k>0 .
\end{aligned}
$$

We wish to write the first two conditions as an equation involving functions on $[ \pm n]$.

Lemma 3.3 Let $\sigma \in S_{n}$ and $j:[ \pm n] \rightarrow[d]$. We have $j(-k)=j(\sigma(k))$ for all $k>0$ if and only if $j=j \circ \sigma \delta \sigma^{-1}$.

Proof Suppose that for $k>0$ we have $j(-k)=j(\sigma(k))$. Then for $k>0$ we have $j \circ \sigma \delta \sigma^{-1}(k)=j\left(-\sigma^{-1}(k)\right)=j(-l)=j(\sigma(l))=j(k)$, where $l=\sigma^{-1}(k)>0$. Also $j \circ \sigma \delta \sigma^{-1}(-k)=j(\sigma(k))=j(-k)$. Thus, $j=j \circ \sigma \delta \sigma^{-1}$.

Now suppose that $j=j \circ \sigma \delta \sigma^{-1}$. For $k>0$, we have that $j(-k)=j \circ \sigma \delta \sigma^{-1}(-k)=$ $j(\sigma(k))$, as claimed.

Lemma 3.4 We have

$$
\begin{aligned}
\mathrm{E}\left(g_{s_{1} t_{1}}^{\left(j_{1}\right)} \cdots g_{s_{n} t_{n}}^{\left(j_{n}\right)} \overline{g_{s_{1} t_{1}}^{\left(j_{-1}\right)}} \cdots \overline{g_{s_{-n} t_{n}}^{\left(j_{-n}\right)}}\right) & = \\
& \left|\left\{\sigma \in S_{n} \mid j=j \circ \sigma \delta \sigma^{-1}, s=s \circ \sigma \delta \sigma^{-1}, t=t \circ \sigma\right\}\right| .
\end{aligned}
$$

Proof By Lemma 3.3 and equation (3.3) we have to count the number of permutations $\sigma$ such that $j=j \circ \sigma \delta \sigma^{-1}$ on the set $\left[d_{1}\right], s=s \circ \sigma \delta \sigma^{-1}$ on the set $\left[d_{2}\right]$, and $t=t \circ \sigma$ on the set $[p]$.

Remark 3.5 In the proposition below we use the notation \# $(\sigma)$ to denote the number of cycles in the cycle decomposition of $\sigma$. If $\sigma$ and $\pi$ are permutations, we let $\sigma \vee \pi$ be the partition obtained by regarding $\sigma$ and $\pi$ as partitions where the blocks of the partition are the cycles of the permutation. Now $\sigma \vee \pi$ denotes the supremum of the two partitions in the lattice of partitions. Recall that the function $\sigma \mapsto \#(\sigma)$ is a central function on $S_{n}$, so $\#\left(\pi^{-1} \sigma \pi\right)=\#(\sigma)$ for all $\pi$ and $\sigma$. 
Proposition 3.6 Subject to the conditions $j=j \circ \epsilon \gamma \delta \gamma^{-1} \epsilon$ and $s=s \circ \eta \gamma \delta \gamma^{-1} \eta$,

$$
\begin{gathered}
\sum_{j_{ \pm 1}, \ldots, j_{ \pm n}} \sum_{s_{ \pm 1}, \ldots, s_{ \pm n}} \sum_{t_{1}, \ldots, t_{n}} \mathrm{E}\left(g_{s_{1} t_{1}}^{\left(j_{1}\right)} \cdots g_{s_{n} t_{n}}^{\left(j_{n}\right)} \overline{g_{s_{-1} t_{1}}^{\left(j_{-1}\right)}} \cdots \overline{g_{s_{-n} t_{n}}^{\left(j_{-n}\right)}}\right)= \\
\sum_{\sigma \in S_{n}} d_{1}^{\#\left(\epsilon \gamma \delta \gamma^{-1} \epsilon \vee \sigma \delta \sigma^{-1}\right)} d_{2}^{\#\left(\eta \gamma \delta \gamma^{-1} \eta \vee \sigma \delta \sigma^{-1}\right)} p^{\#(\sigma) .} .
\end{gathered}
$$

Proof According to Lemma 3.4, subject to the conditions $j=j \circ \epsilon \gamma \delta \gamma^{-1} \epsilon$ and $s=$ $s \circ \eta \gamma \delta \gamma^{-1} \eta$,

$$
\begin{aligned}
& \sum_{j_{ \pm 1}, \ldots, j_{ \pm n}} \sum_{s_{ \pm 1}, \ldots, s_{ \pm n}} \sum_{t_{1}, \ldots, t_{n}} \mathrm{E}\left(g_{s_{1} t_{1}}^{\left(j_{1}\right)} \cdots g_{s_{n} t_{n}}^{\left(j_{n}\right)} \overline{g_{s_{-1} t_{1}}^{\left(j_{-1}\right)}} \cdots \overline{g_{s_{-n} t_{n}}^{\left(j_{-n}\right)}}\right) \\
& =\sum \mid\left\{\sigma \in S_{n} \mid j=j \circ \sigma \delta \sigma^{-1}, s=s \circ \sigma \delta \sigma^{-1} \text {, and } t=t \circ \sigma\right\} \mid \\
& j_{ \pm 1}, \ldots, j_{ \pm n} \\
& s_{ \pm 1}, \ldots, s_{ \pm n} \\
& =\sum_{\sigma \in S_{n}} \mid\left\{(j, s, t) \mid j=j \circ \sigma \delta \sigma^{-1}, s=s \circ \sigma \delta \sigma^{-1} \text {, and } t=t \circ \sigma\right\} \mid \\
& =\sum_{\sigma \in S_{n}} d_{1}^{\#\left(\epsilon \gamma \delta \gamma^{-1} \epsilon \vee \sigma \delta \sigma^{-1}\right)} d_{2}^{\#\left(\eta \gamma \delta \gamma^{-1} \eta \vee \sigma \delta \sigma^{-1}\right)} p^{\#(\sigma)} .
\end{aligned}
$$

To get the last equality we recall that the condition on $j$ is that it must simultaneously satisfy $j=j \circ \epsilon \gamma \delta \gamma^{-1} \epsilon$ and $j=j \circ \sigma \delta \sigma^{-1}$. Then $j$ must be constant on the cycles of $\epsilon \gamma \delta \gamma^{-1} \epsilon$ and of $\sigma \delta \sigma^{-1}$; so $j$ must be constant on the blocks of $\epsilon \gamma \delta \gamma^{-1} \epsilon \vee \sigma \delta \sigma^{-1}$. The same argument applies to $s$. The only condition on $t$ is that $t=t \circ \sigma$.

\section{Theorem 3.7 We have}

$$
\mathrm{E}\left(\operatorname{tr} \otimes \operatorname{tr}\left(W^{\left(\epsilon_{1}, \eta_{1}\right)} \ldots W^{\left(\epsilon_{n}, \eta_{n}\right)}\right)\right)=\sum_{\sigma \in S_{n}}\left(\frac{p}{d_{1} d_{2}}\right)^{\#(\sigma)} d_{1}^{f_{\epsilon}(\sigma)} d_{2}^{f_{\eta}(\sigma)}
$$

where $f_{\epsilon}(\sigma)=\#\left(\epsilon \gamma \delta \gamma^{-1} \epsilon \vee \sigma \delta \sigma^{-1}\right)+\#(\sigma)-(n+1)$ and $f_{\eta}(\sigma)=\#\left(\eta \gamma \delta \gamma^{-1} \eta \vee \sigma \delta \sigma^{-1}\right)+$ $\#(\sigma)-(n+1)$.

Proof According to equation (3.2) and Proposition 3.6 we have

$$
\begin{aligned}
& \mathrm{E}\left(\operatorname{tr} \otimes \operatorname{tr}\left(W^{\left(\epsilon_{1}, \eta_{1}\right)} \cdots W^{\left(\epsilon_{n}, \eta_{n}\right)}\right)\right) \\
& =\left(\frac{1}{d_{1} d_{2}}\right)^{n+1} \sum_{\sigma \in S_{n}} d_{1}^{\#\left(\epsilon \gamma \delta \gamma^{-1} \epsilon \vee \sigma \delta \sigma^{-1}\right)} d_{2}^{\#\left(\eta \gamma \delta \gamma^{-1} \eta \vee \sigma \delta \sigma^{-1}\right)} p^{\#(\sigma)} \\
& =\sum_{\pi \in S_{n}}\left(\frac{p}{d_{1} d_{2}}\right)^{\#(\sigma)} d_{1}^{\#\left(\epsilon \gamma \delta \gamma^{-1} \epsilon \vee \sigma \delta \sigma^{-1}\right)+\#(\sigma)-(n+1)} \\
& \times d_{2}^{\#\left(\eta \gamma \delta \gamma^{-1} \eta \vee \sigma \delta \sigma^{-1}\right)+\#(\sigma)-(n+1)} \\
& =\sum_{\sigma \in S_{n}}\left(\frac{p}{d_{1} d_{2}}\right)^{\#(\sigma)} d_{1}^{f_{\epsilon}(\sigma)} d_{2}^{f_{\eta}(\sigma)} \text {. }
\end{aligned}
$$




\section{Asymptotics of Permutations}

Theorem 3.7 gave us an expansion of mixed moments of $\left\{W, W^{\mathrm{T}}, W^{\mathrm{\Gamma}}, W^{\mathrm{T}}\right\}$ as a sum over the symmetric group. We now have to determine which permutations contribute to the limit. We shall show that for all $\epsilon$ and all $\sigma, f_{\epsilon}(\sigma) \leq 0$ and determine for which $\sigma$ equality is achieved. Our first goal is to show that $f_{\epsilon}(\sigma)<0$ unless $\epsilon$ is constant on the cycles of $\sigma$. Since $\epsilon$ is arbitrary, whatever we show for $\epsilon$ will apply to $\eta$.

There is a fundamental equation that we shall frequently use in what follows. Given a subgroup, $G$, of the group $S_{n}$ of permutations of $[n]$, we shall say that the subgroup acts transitively on $[n]$ if given $k, l \in[n]$ we can find $\rho \in G$ such that $\rho(k)=l$.

Given two permutations $\pi$ and $\sigma$ of $S_{n}$ such that the subgroup generated $\pi$ and $\sigma$ acts transitively, there is a non-negative integer $g$ such that

$$
\#(\pi)+\#\left(\pi^{-1} \sigma\right)+\#(\sigma)=n+2(1-g)
$$

see [10, Theorem 5.9] and [5, p. 14] for a discussion.

Recall that a pairing of $[n]$ is a partition $\pi$ of $[n]$ with all blocks of size 2 ; note this implies that $n$ is even. The set of all pairings of $[n]$ is denoted $\mathcal{P}_{2}(n)$. We shall also regard such a $\pi$ as the permutation whose cycles are the blocks of $\pi$. In this case, $\pi$ has no fixed points and $\pi^{2}=i d$. In [8, Lemma 2] we proved the following lemma.

Lemma 4.1 Let $\pi, \sigma \in \mathcal{P}_{2}(n)$ be pairings and $\left(i_{1}, i_{2}, \ldots, i_{k}\right)$ a cycle of $\pi \sigma$. Let $j_{r}=$ $\sigma\left(i_{r}\right)$. Then $\left(j_{k}, j_{k-1}, \ldots, j_{1}\right)$ is also a cycle of $\pi \sigma$, and these two cycles are distinct; $\left\{i, \ldots, i_{k}, j_{1}, \ldots, j_{k}\right\}$ is a block of $\pi \vee \sigma$ and all are of this form; $2 \#(\pi \vee \sigma)=\#(\pi \sigma)$. The cycle decomposition of $\pi \sigma$ can be written $c_{1} c_{1}^{\prime} \cdots c_{k} c_{k}^{\prime}$ where $c_{i}^{\prime}=\sigma c_{i}^{-1} \sigma$. With this notation, the blocks of $\pi \vee \sigma$ are $c_{i} \cup c_{i}^{\prime}$.

Lemma 4.2 Let $\sigma \in S_{n}$ and $\epsilon \in \mathbb{Z}_{2}^{n}$ be given; then $\epsilon$ is constant on the cycles of $\sigma$ if and only if $\epsilon \delta \sigma \delta \sigma^{-1} \epsilon[n]=[n]$.

Proof We begin by recalling (Remark 3.1) that $\sigma$ acts trivially on $[-n]$ and $\delta \sigma \delta$ acts trivially on $[n]$, and thus noting that for $k>0$

$$
\epsilon \delta \sigma \delta \sigma^{-1} \epsilon(k)=\left\{\begin{array}{ll}
\epsilon \delta \sigma \delta \sigma^{-1}(k) & \epsilon_{k}=1, \\
\epsilon \delta \sigma(k) & \epsilon_{k}=-1,
\end{array}= \begin{cases}\epsilon_{\sigma^{-1}(k)} \sigma^{-1}(k) & \epsilon_{k}=1, \\
-\epsilon_{\sigma(k)} \sigma(k) & \epsilon_{k}=-1 .\end{cases}\right.
$$

Suppose $\epsilon$ is constant on the cycles of $\sigma$. Then

$$
\epsilon \delta \sigma \delta \sigma^{-1} \epsilon(k)=\left\{\begin{array}{ll}
\sigma^{-1}(k) & \epsilon_{k}=1, \\
\sigma(k) & \epsilon_{k}=-1,
\end{array} \in[n] .\right.
$$

Conversely, suppose that $\epsilon \delta \sigma \delta \sigma^{-1} \epsilon[n]=[n]$. Then for $k>0$ by equation (4.2),

$$
\epsilon \delta \sigma \delta \sigma^{-1} \epsilon(k)= \begin{cases}\epsilon_{\sigma^{-1}(k)} \sigma^{-1}(k) & \epsilon_{k}=1, \\ -\epsilon_{\sigma(k)} \sigma(k) & \epsilon_{k}=-1,\end{cases}
$$

and thus $\epsilon_{\sigma(k)}$ and $\epsilon_{k}$ have the same sign.

Lemma 4.3 Let $\sigma \in S_{n}$ and $\epsilon \in \mathbb{Z}_{2}^{n}$ be given; then $f_{\epsilon}(\sigma)<0$ unless $\epsilon$ is constant on the cycles of $\sigma$. 
Proof Suppose $\epsilon$ is not constant on the cycles of $\sigma$; then by Lemma 4.2 we have that $\epsilon \delta \sigma \delta \sigma^{-1} \epsilon[n]$ meets $[-n]$. Both $\epsilon \gamma \delta \gamma^{-1} \epsilon$ and $\sigma \delta \sigma^{-1}$ are pairings and as permutations $\delta$ and $\epsilon$ commute. Thus, by Lemma 4.1,

$$
\begin{aligned}
2 \#\left(\epsilon \gamma \delta \gamma^{-1} \epsilon \vee \sigma \delta \sigma^{-1}\right) & =\#\left(\epsilon \gamma \delta \gamma^{-1} \epsilon \sigma \delta \sigma^{-1}\right)=\#\left(\gamma \delta \gamma^{-1} \delta \epsilon \delta \sigma \delta \sigma^{-1} \epsilon\right) \\
& =\#\left(\left(\epsilon \delta \sigma^{-1} \delta \sigma \epsilon\right)^{-1} \gamma \delta \gamma^{-1} \delta\right) .
\end{aligned}
$$

Now $\#\left(\gamma \delta \gamma^{-1} \delta\right)=2$ and $\#\left(\epsilon \delta \sigma \delta \sigma^{-1} \epsilon\right)=\#\left(\delta \sigma \delta \sigma^{-1}\right)=\#(\delta \sigma \delta)+\#\left(\sigma^{-1}\right)=2 \#(\sigma)$. Hence, by equation (4.1) there is $g \geq 0$ such that

$$
\#\left(\epsilon \delta \sigma^{-1} \delta \sigma \epsilon\right)+\#\left(\left(\epsilon \delta \sigma^{-1} \delta \sigma \epsilon\right)^{-1} \gamma \delta \gamma^{-1} \delta\right)+\#\left(\gamma \delta \gamma^{-1} \delta\right)=2 n+2(1-g),
$$

and thus,

$$
f_{\epsilon}(\sigma)=\#\left(\epsilon \gamma \delta \gamma^{-1} \epsilon \vee \sigma \delta \sigma^{-1}\right)+\#(\sigma)-(n+1)=-(g+1) \leq-1 .
$$

Lemma 4.4 Suppose that $\sigma \in S_{n}$ and $\epsilon \in \mathbb{Z}_{2}^{n}$ and $\epsilon$ is constant on the cycles of $\sigma$. Then there is a permutation $\sigma_{\epsilon} \in S_{n}$ such that $\epsilon \delta \sigma \delta \sigma^{-1} \epsilon=\delta \sigma_{\epsilon} \delta \sigma_{\epsilon}^{-1}$. Moreover, if $\sigma=c_{1} \cdots c_{k}$ is the cycle decomposition of $\sigma$, then $\sigma_{\epsilon}=c_{1}^{\lambda_{1}} \cdots c_{k}^{\lambda_{k}}$, where $\lambda_{i}$ is the (constant) value of $\epsilon$ on the cycle $c_{i}$.

Proof In the proof of Lemma 4.2 we showed that when $\epsilon$ is constant on the cycles of $\sigma$, we have that for $k>0$

$$
\epsilon \delta \sigma \delta \sigma^{-1} \epsilon(k)= \begin{cases}\sigma^{-1}(k) & \text { if } \epsilon_{k}=1 \\ \sigma(k) & \text { if } \epsilon_{k}=-1\end{cases}
$$

Thus on a cycle of $\sigma$ on which $\epsilon=1$ we have $\sigma_{\epsilon}^{-1}$ and $\epsilon \delta \sigma \delta \sigma^{-1} \epsilon$ agree and on a cycle on which $\epsilon=-1$ we have $\sigma_{\epsilon}$ and $\epsilon \delta \sigma \delta \sigma^{-1} \epsilon$ agree.

Definition 4.5 Let $\pi \in S_{n}$ be a permutation of $[n]$ and $\gamma=(1,2,3, \ldots, n)$. We say that $\pi$ is a non-crossing permutation if $\#(\pi)+\#\left(\pi^{-1} \gamma\right)=n+1$. We shall denote by $S_{N C}(n)$ the non-crossing permutations of $[n]$.

Remark 4.6 We have already used the idea of taking a permutation of $[n]$ and regard it as a partition of $[n]$ by using the decomposition of the permutation into disjoint cycles and making these the blocks of a partition. Biane [4] showed that the permutations that satisfy $\#(\pi)+\#\left(\pi^{-1} \gamma\right)=n+1$; i.e., $g=0$ in equation (4.1), are exactly those whose cycles form a non-crossing partition of $[n]$.

Proposition 4.7 Let $\sigma \in S_{n}$ and $\epsilon \in \mathbb{Z}_{2}^{n}$. Suppose that $\epsilon$ is constant on the cycles of $\sigma$. Then $f_{\epsilon}(\sigma) \leq 0$ with equality only if $\sigma_{\epsilon}$ is a non-crossing permutation. 
Proof Let $\epsilon \delta \sigma \delta \sigma^{-1} \epsilon=\delta \sigma_{\epsilon} \delta \sigma_{\epsilon}^{-1}$ as in Lemma 4.4. As in the proof of Lemma 4.3 we have that

$$
\begin{aligned}
f_{\epsilon}(\sigma) & =\frac{1}{2} \#\left(\gamma \delta \gamma^{-1} \delta \epsilon \delta \sigma \delta \sigma^{-1} \epsilon\right)+\#(\sigma)-(n+1) \\
& =\frac{1}{2} \#\left(\gamma \delta \gamma^{-1} \delta \delta \sigma_{\epsilon} \delta \sigma_{\epsilon}^{-1}\right)+\#\left(\sigma_{\epsilon}\right)-(n+1) \\
& =\frac{1}{2} \#\left(\sigma_{\epsilon}^{-1} \gamma \delta \gamma^{-1} \sigma_{\epsilon} \delta\right)+\#\left(\sigma_{\epsilon}\right)-(n+1) \\
& =\#\left(\sigma_{\epsilon}\right)+\#\left(\sigma_{\epsilon}^{-1} \gamma\right)-(n+1) .
\end{aligned}
$$

By equation (4.1), we have $f_{\epsilon}(\sigma) \leq 0$, and, according to Definition 4.5, $\sigma_{\epsilon}$ is a noncrossing permutation if and only if $f_{\epsilon}(\sigma)=0$

Remark 4.8 As an illustration let us consider two examples: $\epsilon \equiv 1$ and $\epsilon \equiv-1$. First suppose $\epsilon \equiv 1$; then $\#\left(\epsilon \gamma \delta \gamma^{-1} \epsilon \vee \sigma \delta \sigma^{-1}\right)=\frac{1}{2} \#\left(\gamma \delta \gamma^{-1} \sigma \delta \sigma^{-1}\right)=\#\left(\sigma^{-1} \gamma\right)$, so $\sigma_{1}=\sigma$ and $f_{1}(\sigma)=\#(\sigma)+\#\left(\sigma^{-1} \gamma\right)-(n+1)=0$ only if $\sigma$ is non-crossing. When $\epsilon \equiv-1$, we have that $\#\left(\epsilon \gamma \delta \gamma^{-1} \epsilon \vee \sigma \delta \sigma^{-1}\right)=\frac{1}{2} \#\left(\delta \gamma \delta \gamma^{-1} \delta \sigma \delta \sigma^{-1}\right)=\#\left(\sigma^{-1} \gamma\right)$, so $\sigma_{1}=\sigma^{-1}$ and $f_{-1}(\sigma)=\#\left(\sigma^{-1}\right)+\#(\sigma \gamma)-(n+1)=0$ only if $\sigma^{-1}$ is non-crossing.

\section{Limit Distributions}

We assume that $d_{1} d_{2} \rightarrow \infty$ and that $\frac{p}{d_{1} d_{2}} \rightarrow c$, for some $0<c<\infty$. Since $W$ and $W^{\mathrm{T}}$ are Wishart matrices, their eigenvalue distributions converge to the MarchenkoPastur law with parameter $c$. Setting $b=(1+\sqrt{c})^{2}$ and $a=(1-\sqrt{c})^{2}$, this is the distribution on $\mathbb{R}^{+}$that has density $\sqrt{(b-t)(t-a)} / 2 \pi t$ on $[a, b]$ and an atom of weight $(1-c)$ at 0 if $c<1$.

The asymptotic eigenvalue distributions of $W^{\mathrm{T}}$ and $W^{\mathrm{T}}$ were described by G. Auburn (see [2]) for the case when $d_{1}, d_{2} \rightarrow \infty$, respectively by T. Banica and I. Nechita for the case when $d_{2} \rightarrow \infty$ (see [3]). The calculations below give another proof of these results and give some more insight on the limit distributions.

Lemma 5.1 Let $\sigma \in S_{n}$ and suppose that both $\sigma$ and $\sigma^{-1}$ are non-crossing in the sense of Definition 4.5. Then $\sigma$ can have only cycles of size 1 or 2 .

Before proving Lemma 5.1, we need to recall some standard facts about permutations and pairings. We recall that $[ \pm n]=\{1,-1,2,-2, \ldots, n,-n\}$. If $\sigma \in S_{n}$ is a permutation of $[n]$, then $\sigma \delta \sigma^{-1}$ is a pairing of $[ \pm n]$; moreover if $(r, s)$ is a pair in this pairing, then $r$ and $s$ have opposite signs. We let $\mathcal{P}_{2}^{\delta}( \pm n)$ be the set of pairings of $[ \pm n]$ that only pair a positive number to a negative number. There is a standard bijection from $S_{n}$ to $\mathcal{P}_{2}^{\delta}( \pm n)$ that we will use. For $\sigma \in S_{n}$ we have $\sigma \delta \sigma^{-1} \in \mathcal{P}_{2}^{\delta}( \pm n)$. If $\pi \in \mathcal{P}_{2}^{\delta}( \pm n)$ then $\pi \delta$ leaves $[n]$ invariant and so $\left.\pi \delta\right|_{[n]} \in S_{n}$. These two maps are inverses of each other.

For example, consider $\gamma=(1,2, \ldots, n) \in S_{n}$. Then

$\gamma \delta \gamma^{-1}=(-n, 1)(-1,2)(-2,3) \cdots(-(n-1), n) \in \mathcal{P}_{2}^{\delta}( \pm n) \quad$ and $\left.\quad\left(\gamma \delta \gamma^{-1}\right) \delta\right|_{[n]}=\gamma$.

Also the permutation $\gamma \delta$ has the one cycle $(1,-1,2,-2, \ldots, n,-n)$.

Inside $\mathcal{P}_{2}^{\delta}( \pm n)$ we have the non-crossing pairings of $[ \pm n]$, which only connect a positive number to a negative number; we shall denote this subset by $N C_{2}^{\delta}( \pm n)$. 
Lemma 5.2 The map $\sigma \mapsto \sigma \delta \sigma^{-1}$ is a bijection from $S_{N C}(n)$ to $N C_{2}^{\delta}( \pm n)$.

Proof We have to check that $\sigma \in S_{N C}(n)$ if and only if $\sigma \delta \sigma^{-1} \in N C_{2}^{\delta}( \pm n)$. Note that $\sigma \delta \sigma^{-1}$ is a pairing so that $\#\left(\sigma \delta \sigma^{-1}\right)=n$. Also $\#\left(\left(\sigma \delta \sigma^{-1}\right)^{-1} \gamma \delta\right)=\#\left(\delta \sigma \delta \sigma^{-1} \gamma\right)=$ $\#(\delta \sigma \delta)+\#\left(\sigma^{-1} \gamma\right)=\#(\sigma)+\#\left(\sigma^{-1} \gamma\right)$, because $\delta \sigma \delta$ acts trivially on $[n]$ and $\sigma^{-1} \gamma$ acts trivially on $[-n]$ (cf. Remark 3.1). Thus, $\#\left(\sigma \delta \sigma^{-1}\right)+\#\left(\left(\sigma \delta \sigma^{-1}\right)^{-1} \gamma \delta\right)=n+\#(\sigma)+$ $\#\left(\sigma^{-1} \gamma\right)$. By Remark 4.6 we have that $\sigma$ is non-crossing if and only if $\sigma \delta \sigma^{-1}$ is noncrossing.

Proof of Lemma 5.1 Suppose that $\sigma \in S_{N C}(n)$ and $i_{1}<i_{2}<i_{3}$ are distinct with $\sigma\left(i_{1}\right)=i_{2}$ and $\sigma\left(i_{2}\right)=i_{3}$. Then $\gamma \delta$ visits $\left\{i_{1},-i_{1}, i_{2},-i_{2}, i_{3},-i_{3}\right\}$ and $\left(i_{1},-i_{2}\right)$ and $\left(i_{2},-i_{3}\right)$ are pairs of $\sigma^{-1} \delta \sigma$. Thus, $\sigma^{-1} \delta \sigma$ is not in $N C_{2}^{\delta}( \pm n)$, and hence by Lemma 5.2 $\sigma^{-1} \notin S_{N C}(n)$. Thus, the only permutations $\sigma \in S_{N C}(n)$ for which $\sigma^{-1} \in S_{N C}(n)$ are those where $\sigma=\sigma^{-1}$; i.e., all cycles are singletons or pairs.

Theorem 5.3 ([2, Thm. 1]) If $d_{1}, d_{2} \rightarrow \infty$, then the limit distributions of $W^{\mathrm{T}}$ and $W^{\Gamma}$ are semi-circular with mean $c$ and variance $c$.

Proof We let $\epsilon \equiv 1$ and $\eta \equiv-1$; then $W^{\left(\epsilon_{1}, \eta_{1}\right)} \ldots W^{\left(\epsilon_{n}, \eta_{n}\right)}=\left(W^{\Gamma}\right)^{n}$. Also $f_{\epsilon}(\sigma)=$ $f_{1}(\sigma)=\#\left(\sigma^{-1} \gamma\right)+\#(\sigma)-(n+1)$ and $f_{\eta}(\sigma)=f_{-1}(\sigma)=f_{1}\left(\sigma^{-1}\right)$. So by equation (3.4) we have

$$
\mathrm{E}\left(\operatorname{tr}\left(\left(W^{\Gamma}\right)^{n}\right)=\sum_{\sigma \in S_{n}}\left(\frac{p}{d_{1} d_{2}}\right)^{\#(\sigma)} d_{1}^{f_{1}(\sigma)} d_{2}^{f_{1}\left(\sigma^{-1}\right)} .\right.
$$

Thus, by Definition 4.5 and Lemma 5.1, the only $\sigma$ 's that contribute to the limit are those for which $\sigma$ is non-crossing and has only blocks of size 1 or 2 . Let us denote the set of such $\sigma$ 's by $N_{1,2}(n)$. We have shown that the moments of $W^{\Gamma}$ converge to those of an element $w^{\Gamma}$ in a non-commutative probability space $(\mathcal{A}, \varphi)$ with

$$
\varphi\left(\left(w^{\Gamma}\right)^{n}\right)=\sum_{\sigma \in N C_{1,2}(n)} c^{\#(\sigma)} .
$$

By the moment-cumulant formula [10, Definition 2.8], we have just computed the cumulants of $w^{\Gamma}$. Moreover, we have shown that the only non-vanishing cumulants of $w^{\Gamma}$ are $\kappa_{1}=\kappa_{2}=c$. Thus, the limiting distribution is semi-circular.

Remark 5.4 The measure on $\mathbb{R}$ whose free cumulants are $\kappa_{1}=\kappa_{2}=c$ and $\kappa_{n}=0$ for $n \geq 3$ is the shifted semi-circle law. It has density $\frac{1}{2 \pi c} \sqrt{4 c^{2}-(t-c)^{2}}$ on the interval $[c-2 \sqrt{c}, c+2 \sqrt{c}]$. We have used a different normalization for $W$ than Aubrun (we used $\frac{1}{d_{1} d_{2}}$ and he used $\frac{1}{p}$ ); the advantage of ours is that the free cumulants are very simple with this normalization.

Next, we discuss the case when only one of the parameters $d_{1}, d_{2}$ approaches infinity and the other one is fixed.

The following remarkable result is due to T. Banica and I. Nechita [3, Lemma 1.1].

Lemma 5.5 Suppose that $\sigma$ is a non-crossing permutation and that $\tau$ is a cycle of length $n$ in $S_{n}$. Then $\#(\sigma \tau)=1+e(\sigma)$, where $e(\sigma)$ is the number of cycles of $\sigma$ of even length. 
Let us recall the main result of [3, Theorem 3.1], which computes the free cumulants of the limit distribution of $d_{1} W^{\mathrm{T}}$ as $p /\left(d_{1} d_{2}\right) \rightarrow c$ while keeping $d_{1}$ fixed.

Theorem 5.6 Suppose that $d_{1}$ is a fixed positive integer, and $\frac{p}{d_{1} d_{2}} \rightarrow c$ with $0<c<\infty$. The free cumulants of the limit distribution of $d_{1} W^{\mathrm{T}}$ are $\kappa_{n}=c d_{1}^{2}$ for $n$ even and $\kappa_{n}=$ $c d_{1}$ for $n$ odd. This limit distribution is the free difference of two Marchenko-Pastur laws, one with parameter $c d_{1} \frac{d_{1}+1}{2}$ and the other $c d_{1} \frac{d_{1}-1}{2}$.

Proof Let $\epsilon \equiv 1$ and $\eta \equiv-1$ in Theorem 3.7. By Remark 4.8, $f_{\epsilon}(\sigma)<0$ unless $\sigma \in N C(n)$. For $\sigma \in N C(n)$ and $\eta \equiv-1$ we have by Remark 4.8 and Lemma 5.5, $f_{\eta}(\sigma)-\#(\sigma)+n=e(\sigma)$. Hence Theorem 3.7 gives

$$
\lim _{d_{2} \rightarrow \infty} \mathrm{E}\left(\operatorname{tr} \otimes \operatorname{tr}\left(\left(d_{1} W^{\mathrm{T}}\right)^{n}\right)\right)=\sum_{\sigma \in N C(n)} c^{\#(\sigma)} d_{1}^{f_{-1}(\sigma)+n}=\sum_{\sigma \in N C(n)}\left(d_{1} c\right)^{\#(\sigma)} d_{1}^{e(\sigma)} .
$$

Note that if we set $\kappa_{n}=d_{1}^{2} c$ when $n$ is even and $\kappa_{n}=d_{1} c$ when $n$ is odd, then $\kappa_{\sigma}=\left(d_{1} c\right)^{\#(\sigma)} d_{1}^{e(\sigma)}$. This shows that the limit distribution of $d_{1} W^{\mathrm{T}}$ has the claimed cumulants. Since $\kappa_{n}=\left(d_{1} c\right) \frac{d_{1}+1}{2}+(-1)^{n}\left(d_{1} c\right) \frac{d_{1}-1}{2}$, we have the claim about the distribution being a free difference of Marchenko-Pastur laws.

Remark 5.7 If in Theorem 3.7 we let $\epsilon \equiv-1$ and $\eta \equiv 1$, the coefficients $d_{1}$ and $d_{2}$ switch roles, hence the argument above also gives an analogous statement for holding $d_{2}$ fixed. More precisely, if $d_{2}$ is fixed and $p /\left(d_{1} d_{2}\right) \rightarrow c$, then the free cumulants of the limit distribution of $d_{2} W^{\mathrm{\Gamma}}$ are given by $\kappa_{n}=d_{2}^{2} c$ for $n$ even and $\kappa_{n}=d_{2} c$ for $n$ odd. This distribution is also the free difference of two Marchenko-Pastur distributions one of parameter $d_{2} c \frac{d_{2}+1}{2}$ and one of $d_{2} c \frac{d_{2}-1}{2}$.

Remark 5.8 Since taking transposes preserves eigenvalue distributions Theorem 5.6 and Remark 5.7 also gives us the free cumulants of the limit distribution of $d_{1} W^{\mathrm{\Gamma}}$ and $d_{2} W^{\mathrm{T}}$.

\section{A Natural Free Decomposition of $d_{1} W^{\mathrm{T}}$ when $d_{1}=2$}

In [3] it was shown that the limit distribution of $d_{1} W^{\mathrm{T}}$ can be written as the free difference of two Marchenko-Pastur laws. The operators so obtained are not related to the operator $d_{1} W^{\mathrm{T}}$, though. In this section we shall show that there is a natural decomposition of $d_{1} W^{\mathrm{T}}$ when $d_{1}=2$, namely the diagonal and off diagonal blocks, into free summands. More precisely, we let $w$ be the limit distribution of $W$, which we can write $w$ as a $2 \times 2$ matrix

$$
w=\frac{1}{2}\left(\begin{array}{ll}
w_{11} & w_{12} \\
w_{21} & w_{22}
\end{array}\right) .
$$

Relative to this block decomposition, $2 W^{\mathrm{T}}$ converges to

$$
2 w^{\mathrm{T}}=\left(\begin{array}{ll}
w_{11} & w_{21} \\
w_{12} & w_{22}
\end{array}\right) .
$$


We consider the two operators

$$
X_{1}=\left(\begin{array}{cc}
w_{11} & 0 \\
0 & w_{22}
\end{array}\right) \quad \text { and } \quad X_{2}=\left(\begin{array}{cc}
0 & w_{21} \\
w_{12} & 0
\end{array}\right) .
$$

The diagonal summand $X_{1}$ has the Marchenko-Pastur distribution and the off diagonal summand $X_{2}$ is even and has the same even cumulants as the diagonal summand. Our main result in this section is that $X_{1}$ and $X_{2}$ are free.

Notation 6.1 Let $d_{1}=2$, and suppose $p /\left(d_{1} d_{2}\right)$ converges to $c$ with $0<c<\infty$. Let $\left\{E_{11}, E_{12}, E_{21}, E_{22}\right\}$ be the standard matrix units in $M_{2}(\mathbb{C})$, but viewed as elements of $M_{2}(\mathbb{C}) \otimes M_{d_{2}}(\mathbb{C})$.

Lemma 6.2 There is a *-non-commutative probability space $(\mathcal{A}, \varphi)$ with elements $w, e_{11}, e_{12}, e_{21}, e_{22} \in \mathcal{A}$ such that $w$ has the Marchenko-Pastur distribution with parameter $c$ and $\left\{e_{11}, e_{12}, e_{21}, e_{22}\right\}$ are matrix units in $\mathcal{A}$ free from $w$. Moreover, the joint distribution of $\left\{W, E_{11}, E_{12}, E_{21}, E_{22}\right\}$ converges to that of $\left\{w, e_{11}, e_{12}, e_{21}, e_{22}\right\}$.

Proof As $W$, our Wishart matrix, is unitarily invariant, it is asymptotically free from our matrix units (see [10, Theorem 4.9]). This is exactly the claim of the lemma.

Notation 6.3 Thus, we can write the matrix of $w$ with respect to the matrix units $\left\{e_{11}, e_{12}, e_{21}, e_{22}\right\}$ as

$$
w=\frac{1}{2}\left(\begin{array}{ll}
w_{11} & w_{12} \\
w_{21} & w_{22}
\end{array}\right)
$$

We will let $\varphi_{1}$ be the state on $e_{11} \mathcal{A} e_{11}$ given by $\varphi_{1}(x)=2 \varphi(x)$. The elements $\left\{w_{11}, w_{12}, w_{21}, w_{22}\right\}$ are in $e_{11} \mathcal{A} e_{11}$, so their cumulants must be computed relative to the state $\varphi_{1}$. When necessary, we will denote these relative cumulants by $\kappa_{n}^{(1)}$.

Lemma 6.4 Each of $w_{11}$ and $w_{22}$ has the Marchenko-Pastur distribution with parameter $d_{1} c$.

Proof By construction, $w_{11}=e_{11} 2 w e_{11}$. By [11, Theorem 14.18],

$$
\kappa_{n}^{(1)}\left(w_{11}, \ldots w_{11}\right)=2^{n} \kappa_{n}\left(e_{11} w e_{11}, \ldots, e_{11} w e_{11}\right)=2^{1} \kappa_{n}(w, \ldots, w)=2 c .
$$

Remark 6.5 Elements $\left\{a_{i j}\right\}_{i, j=1}^{n}$ in a non-commutative probability space $(\mathcal{A}, \varphi)$, they are called $R$-cyclic if, whenever $i_{1}, j_{1}, \ldots, i_{l}, j_{l} \in[n]$, we have $\kappa_{l}\left(a_{i_{1} j_{1}}, \ldots, a_{i_{l} j_{l}}\right)=$ 0 unless $j_{1}=i_{2}, \ldots, j_{n-1}=i_{n}$ and $j_{n}=i_{1}$. By [11, Example 20.4], the elements $\left\{w_{11}, w_{12}, w_{21}, w_{22}\right\}$ are $R$-cyclic. Moreover, $\kappa_{l}(2 w, \ldots, 2 w)=2^{l} \kappa_{l}(w, \ldots, w)=2^{l} c$. So by [11, Example 20.4], we have $\kappa_{l}^{(1)}\left(w_{i_{1} j_{1}}, w_{i_{2} j_{2}}, \ldots, w_{i_{l} j_{l}}\right)=2^{-l+1} \kappa_{l}(2 w, \ldots, 2 w)=$ $2 c$, when $j_{1}=i_{2}, \ldots, j_{n-1}=i_{n}$ and $j_{n}=i_{1}$.

$$
\text { Let } X_{1}=\left(\begin{array}{cc}
w_{11} & 0 \\
0 & w_{22}
\end{array}\right) \text { and } X_{2}=\left(\begin{array}{cc}
0 & w_{21} \\
w_{12} & 0
\end{array}\right) \text {. Then } 2 w^{\mathrm{T}}=X_{1}+X_{2} \text {. }
$$

Lemma 6.6 $X_{1}$ and $X_{2}$ are self-adjoint. The cumulants of $X_{1}$ are all equal to $2 c$; i.e., $X_{1}$ is a Marchenko-Pastur operator with parameter $2 c$, and $X_{2}$ is an even operator in 
that it is self-adjoint and all of its odd moments are 0 . The even cumulants of $X_{2}$ are all equal to $2 c$.

Proof We have $\varphi\left(X_{1}^{l}\right)=\varphi^{(1)}\left(w_{11}^{l}\right)$, so $X_{1}$ and $w_{11}$ have the same cumulants, which by Remark 6.5 are all $2 c$. Because $X_{2}$ is off diagonal and self-adjoint, it is an even operator. By [11, Proposition 15.12] the cumulants of $X_{2}$ are the $*$-cumulants of $w_{21}$. In Remark 6.5, we observed that these are all $2 c$.

Our next goal is to show that $X_{1}$ and $X_{2}$ are free in $(\mathcal{A}, \varphi)$. This is somewhat surprising in that $X_{1}$ and $X_{2}^{\mathrm{T}}$ are not free. By $X_{2}^{\mathrm{T}}$, we mean the matrix $\left(\begin{array}{cc}0 & w_{12} \\ w_{21} & 0\end{array}\right)$. To see this note that $\varphi\left(X_{1} X_{2}^{\mathrm{T}} X_{2}^{\mathrm{T}} X_{1}\right)=2 c+3(2 c)^{2}+(2 c)^{3}$, whereas if $X_{1}$ and $X_{2}$ were free, we would have $\varphi\left(X_{1} X_{2}^{\mathrm{T}} X_{2}^{\mathrm{T}} X_{1}\right)=(2 c)^{2}+(2 c)^{3}$. This gives another unexpected instance where a partial transpose produces freeness, but this time at the level of operators.

Now let us turn to the freeness of $X_{1}$ and $X_{2}$. Let

$$
Y_{1}=X_{1}, Y_{2}=\left(\begin{array}{cc}
w_{21}, & 0 \\
0 & w_{12}
\end{array}\right) \text { and } Y_{3}=\left(\begin{array}{ll}
0 & 1 \\
1 & 0
\end{array}\right) \text {. }
$$

Then $X_{2}=Y_{2} Y_{3}$. Let $i_{1}, \ldots, i_{n}$ be in [3]. We let $\operatorname{ker}(i)$ be the partition of $[n]$ such that $r$ and $s$ are in the same block of $\operatorname{ker}(i)$ if and only if $i_{r}=i_{s}$. We let $1_{n}$ be the partition of $[n]$ with one block. Then $\operatorname{ker}(i)=1_{n}$ if and only if $i$ is the constant function. Suppose that $\operatorname{ker}(i)<1_{n}$. Let $r$ be the number of times $i=2$, and $q=n+r$. Then there are $j_{1}, j_{2}, \ldots, j_{q} \in\{1,2,3\}$ such that $X_{i_{1}} \cdots X_{i_{n}}=Y_{j_{1}} \cdots Y_{j_{q}}$. We now apply the formula for cumulants with products as entries [11, Theorem 11.12]. Then

$$
\kappa_{n}\left(X_{i_{1}}, \ldots, X_{i_{n}}\right)=\sum_{\pi \in N C(q)} \kappa_{\pi}\left(Y_{j_{1}}, \ldots, Y_{j_{q}}\right),
$$

where the sum runs over all non-crossing partitions in $N C(q)$ such that $\pi \vee \rho=1_{q}$ and $\rho$ is the non-crossing partition whose blocks are have either 1 or 2 elements, and the singletons are where $j_{l}=1$ appears in the string $Y_{j_{1}} \cdots Y_{j_{q}}$, and the pairs are $(l, l+1)$ where $j_{l}=2$ and $j_{l+1}=3$. Since $\operatorname{ker}(i)<1_{n}$, there must both singletons and pairs. Let us consider a $\pi \in N C(q)$ that is such that $\pi \vee \rho=1_{q}$; we will show that by the $R$-cyclicity of $w$, we have $\kappa_{\pi}\left(Y_{j_{1}}, \ldots, Y_{j_{q}}\right)=0$. Summing over all such $\pi$, we get that $\kappa_{n}\left(X_{i_{1}}, \ldots, X_{i_{n}}\right)=0$. This shows that all mixed cumulants vanish and hence that $X_{1}$ and $X_{2}$ are free, as claimed.

Lemma 6.7 Given $p_{1}, \ldots, p_{s} \in[3]$, we have $\varphi\left(Y_{p_{1}} \cdots Y_{p_{s}}\right)=0$ unless $Y_{3}$ appears an even number of times.

Proof $Y_{1}$ and $Y_{2}$ are diagonal, so $Y_{p_{1}} \cdots Y_{p_{s}}$ will be 0 on the diagonal unless $Y_{3}$ appears an even number of times.

Lemma 6.8 Given $p_{1}, \ldots, p_{s} \in[3]$, we have $\kappa_{s}\left(Y_{p_{1}}, \ldots, Y_{p_{s}}\right)=0$ unless $Y_{3}$ appears an even number of times.

Proof We write

$$
\kappa_{s}\left(Y_{p_{1}}, \ldots, Y_{p_{s}}\right)=\sum_{\pi \in N C(s)} \mu\left(\pi, 1_{s}\right) \varphi_{\pi}\left(Y_{p_{1}}, \ldots, Y_{p_{s}}\right)
$$


Given $\pi$, we have by Lemma 6.7 that each block of $\pi$ must contain an even number of $Y_{3}$ 's, or else $\varphi_{\pi}\left(Y_{p_{1}}, \ldots, Y_{p_{s}}\right)=0$. Summing over all blocks of $\pi$, we get that the number of $Y_{3}$ 's is even.

Definition 6.9 Let $i_{1}, \ldots, i_{s} \in[3]$, we say that the $s$-tuple has the property $(n v c)$ if each non-zero entry of $Y_{i_{1}} \cdots Y_{i_{s}}$ is of the form $w_{u_{1} v_{1}} \cdots w_{u_{k} v_{k}}$, where $v_{1}=u_{2}, v_{2}=$ $u_{3}, \ldots, v_{k-1}=v_{k}$. Note that we do not require $v_{k}=u_{1}$ as in $R$-cyclicity. We say that the string has property $(v c)$ if it does not have property $(n c v)$.

Remark 6.10 We now describe the generic sequences with property $(n v c)$. First, we have any power of $Y_{1}$. The product of two or more $Y_{2}$ 's does not have property $(n v c)$. No power of $Y_{3}$ has property $(n v c)$, because all its entries are either 0 or 1 .

Now suppose we start with a $Y_{2}$. We can only follow with a $Y_{1}$ or a $Y_{3}$. So our basic reduced sequence is (with the possibility that $k=0$ )

$$
Y_{2} \underbrace{Y_{1} \cdots Y_{1}}_{k} Y_{3} Y_{2} .
$$

We can enhance this by putting an even power of $Y_{3}$ between any two letters above. Note that there cannot be an odd number of $Y_{3}$ 's between two $Y_{1}$ 's, as

$$
Y_{1} Y_{3} Y_{1}=\left(\begin{array}{cc}
0 & w_{11} w_{22} \\
w_{22} w_{11} & 0
\end{array}\right) .
$$

So the most general string starting and ending with a $Y_{2}$ is

$$
Y_{2} Y_{3}^{l_{1}} Y_{1}^{k_{1}} Y_{3}^{l_{2}} Y_{1}^{k_{2}} \cdots Y_{1}^{k_{r}} Y_{3}^{l_{r+1}} Y_{2}
$$

with $l_{1}, \ldots, l_{r}$ even and $l_{r+1}$ odd.

Lemma 6.11 Let $i_{1}, \ldots, i_{k}$ be a string with property $(n v c)$ that starts and ends with $Y_{2}$ and has no other $Y_{2}$ 's. Then the number of $Y_{3}$ 's is odd.

Proof We just observed that the number of $Y_{3}$ 's is $l_{1}+\cdots+l_{r}+l_{r+1}$, which is odd.

Lemma 6.12 If $\pi \in N C(q)$ and $\pi \vee \rho=1_{q}$ and $\kappa_{\pi}\left(Y_{i_{1}}, \ldots, Y_{i_{q}}\right) \neq 0$, then each block of $\pi$ must contain the same number of $Y_{2}$ 's as $Y_{3}$, and both numbers are even.

Proof We have just observed that the number of $Y_{3}$ 's between $Y_{2}$ 's is odd. Thus, to go all the way round a cycle, the number of $Y_{3}$ 's is equal to the number of $Y_{2}$ 's plus an even number that might be 0 . However, in our whole string the number of $Y_{2}$ 's and $Y_{3}$ 's is the same. If one cycle had an excess of $Y_{3}$ 's, then another cycle would have a deficit. Thus, all cycles must be balanced. Since we already know that each cycle has an even number of $Y_{3}$ 's it also has an equal even number of $Y_{2}$ 's.

Lemma 6.13 Let $i_{1}, \ldots, i_{q} \in[3]$ be such that $\operatorname{ker}(i)<1_{q}$ and let $\pi \in N C(q)$ be such that $\pi \vee \rho=1_{q}$. Then $\kappa_{\pi}\left(Y_{i_{1}}, \ldots, Y_{i_{k}}\right)=0$.

Proof Let $V$ be a block of $\pi$ that contains a $l$ such that $j_{l}=1$. Then $(l)$ is a block of $\rho$. Since we are assuming that $\pi \vee \rho=1_{q}$, there must be $l_{0} \in V$ with $j_{l_{0}} \in\{2,3\}$. If the contribution of this block to $\kappa_{\pi}\left(Y_{i_{1}}, \ldots, Y_{i_{k}}\right)$ is not 0 , then there must be a $Y_{1}$ 
followed by a $Y_{3}$. So we can assume that we have a $l$ and $l^{\prime}$ such that $j_{l}=1, j_{l^{\prime}}=3$, and $l^{\prime}$ follows $l$ in $V$. We have that $\pi$ restricts to a non-crossing partition of $\left[l+1, l^{\prime}-1\right]$. Each block in this restriction contains the same number of $Y_{2}$ 's as $Y_{3}$ 's by Lemma 6.12. However, this is impossible, because in the original string $Y_{i_{1}}, \ldots, Y_{i_{k}}$, a $Y_{2}$ is always followed by a $Y_{3}$, and we have removed one $Y_{3}$. Thus, $\kappa_{\pi}\left(Y_{i_{1}}, \ldots, Y_{i_{k}}\right)=0$.

Theorem $6.14 \quad X_{1}$ and $X_{2}$ are free in $(\mathcal{A}, \varphi)$.

Proof We have just shown that by the formula for cumulants with products for entries we have that mixed cumulants vanish. Thus, $X_{1}$ and $X_{2}$ are free.

Remark 6.15 The distribution of $w^{\mathrm{T}}$ in $(\mathcal{A}, \varphi)$ is the limit distribution of $W^{\mathrm{T}}$ that is the same as $W^{\Gamma}$. Thus, the distribution of $d_{1} w^{\Gamma}$ is the same as that of $d_{1} w^{\mathrm{T}}$.

Theorem 6.16 For $d_{1}=2$ and $p /\left(d_{1} d_{2}\right) \rightarrow c$, the limit distribution of $2 W^{\mathrm{T}}$ is the free additive convolution of a Marchenko-Pastur law with parameter $2 c$ and an even operator with all even cumulants equal to $2 c$.

\section{Asymptotic Freeness}

Since $W$ is unitarily invariant, a consequence of the results from [9] is that $W$ and $W^{T}$ are asymptotically free if $d_{1} d_{2} \rightarrow \infty$. In this section we will present the main results of the paper, which, using the relation form Theorem 3.7, improves the result mentioned above.

Theorem 7.1 If $d_{1} \rightarrow \infty$ and $d_{2} \rightarrow \infty$, then the family $\left\{W, W^{T}, W^{\mathrm{T}}, W^{\mathrm{T}}\right\}$ is asymptotically free.

Proof By Theorem 3.7, we have that

$$
\mathrm{E}\left(\operatorname{tr} \otimes \operatorname{tr}\left(W^{\left(\epsilon_{1}, \eta_{1}\right)} \cdots W^{\left(\epsilon_{n}, \eta_{n}\right)}\right)\right)=\sum_{\sigma \in S_{n}}\left(\frac{p}{d_{1} d_{2}}\right)^{\#(\sigma)} d_{1}^{f_{\epsilon}(\sigma)} d_{2}^{f_{\eta}(\sigma)},
$$

and by Lemma 4.3 and Proposition 4.7, we have that

- $f_{\epsilon}(\sigma), f_{\eta}(\sigma) \leq 0$ for all $\sigma, \epsilon$, and $\eta$;

- $f_{\epsilon}(\sigma), f_{\eta}(\sigma)<0$ unless $\epsilon$ and $\eta$ are constant on the cycles of $\sigma$;

- $f_{\epsilon}(\sigma)<0$ unless $\sigma_{\epsilon}$ is non-crossing.

Thus, when $d_{1}, d_{2} \rightarrow \infty$ and $\frac{p}{d_{1} d_{2}} \rightarrow c$, we need only consider $\sigma$ 's for which

(a) $\epsilon$ and $\eta$ are constant on the cycles of $\sigma$;

(b) both $\sigma_{\epsilon}$ and $\sigma_{\eta}$ are non-crossing.

Note that, as partitions, $\sigma, \sigma_{\epsilon}$, and $\sigma_{\eta}$ are the same, since the only possible difference between them is whether we reverse the order of elements in a cycle of $\sigma$. Thus, we have shown that the limit when $d_{1}, d_{2} \rightarrow \infty$ of an arbitrary mixed moment can be written as a sum over non-crossing partitions; that means that the terms that appear are the free cumulants of the mixed moment we are considering. However, by (a), the blocks of $\sigma$ only connect $W^{\left(\epsilon_{i}, \eta_{i}\right)}$ to $W^{\left(\epsilon_{j}, \eta_{j}\right)}$ if $\left(\epsilon_{i}, \eta_{i}\right)=\left(\epsilon_{j}, \eta_{j}\right)$. This means we have shown that mixed cumulants vanish and this implies the conclusion. 
Theorem $7.2 \quad$ (i) If $d_{1} \rightarrow \infty$ and $d_{2}$ is fixed, then the family $\left\{W, W^{\mathrm{\Gamma}}\right\}$ is asymptotically free from the family $\left\{W^{T}, W^{\mathrm{T}}\right\}$, but $W$ is not asymptotically free from $W^{\mathrm{T}}$, nor is $W^{T}$ from $W^{\mathrm{T}}$.

(ii) If $d_{1}$ is fixed and $d_{2} \rightarrow \infty$, then the family $\left\{W, W^{\mathrm{T}}\right\}$ is asymptotically free from the family $\left\{W^{T}, W^{\mathrm{\Gamma}}\right\}$ but $W$ is not asymptotically free from $W^{\mathrm{\Gamma}}$, nor is $W^{T}$ from $W^{\mathrm{\Gamma}}$.

Proof Suppose first that $d_{1} \rightarrow \infty$ and $d_{2}$ is fixed. Hence, in the summation from Theorem 3.7 only the terms where $f_{\varepsilon}(\sigma)=0$ will contribute to the limit. As in the proof of Theorem 7.1, the last condition is equivalent to $\sigma_{\varepsilon}$ being non-crossing and $\varepsilon$ being constant on the cycles of $\sigma$. Since the partitions $\sigma$ and $\sigma_{\varepsilon}$ are the same, it follows that the limit as $d_{1} \rightarrow \infty$ of an arbitrary mixed moment is written as a sum over noncrossing partitions, so the terms in the right-hand side are in fact free cumulants. The condition that $\varepsilon$ is constant on the cycles of $\sigma$ gives that only free cumulants in elements from only one of the families from part (i) do not vanish, hence the asymptotic freeness is proved.

For the second part of (i), we will use the expansion of $E \circ \operatorname{tr} \otimes \operatorname{tr}\left(W \cdot W^{\mathrm{\Gamma}}\right)$ from Theorem 3.7 in the case $\varepsilon=(1,1)$ and $\eta=(1,-1)$. Note that $S_{2}$ contains only two permutations, $\gamma=(1,2)$ and $\sigma=(1),(2)$, both non-crossing. Also, since $\varepsilon$ is constant, it is constant on the cycles of $\sigma$ and $\gamma$. It follows that $f_{\varepsilon}(\sigma)=f_{\varepsilon}(\gamma)=0$. Moreover, $\eta$ is constant on the cycles of $\sigma$ and $\sigma_{\eta}=\sigma$ is non-crossing, hence $f_{\eta}(\sigma)=0$. Therefore, Theorem 7.1 gives that

$$
E \circ \operatorname{tr} \otimes \operatorname{tr}\left(W \cdot W^{\mathrm{T}}\right)=c^{2}+c \cdot d_{2}^{f_{\eta}(\gamma)} .
$$

As $d_{1} \rightarrow \infty$, the first moment of $W$ approaches $c$, and from Theorem 5.6, so does the first moment of $W^{\mathrm{\Gamma}}$, hence

$$
\lim _{d_{1} \rightarrow \infty} \kappa_{2}\left(W, W^{\Gamma}\right)=c \cdot d_{2}^{f_{\eta}(\gamma)} \neq 0 .
$$

The same argument also shows that $W^{T}$ and $W^{\mathrm{T}}$ are not asymptotically free, since $W^{\mathrm{T}}=\left(W^{\mathrm{T}}\right)^{T}$.

Finally, part (ii) also follows from the argument for part (i), since the relation from Theorem 3.7 is symmetric in $\left(d_{1}, \varepsilon\right)$ and $\left(d_{2}, \eta\right)$.

\section{The Case of Real Wishart Matrices}

In this section we examine the case of real Wishart matrices. More precisely, $W$ will denote now the symmetric $d_{1} d_{2} \times d_{1} d_{2}$ random matrix

$$
W=\frac{1}{d_{1} d_{2}}\left(G_{i} G_{j}^{*}\right)_{i, j=1}^{d_{1}},
$$

where $\left\{G_{i}: 1 \leq i \leq d_{1}\right\}$ is a family of $d_{2} \times p$ random matrices whose entries are independent Gaussian random variables of mean 0 and variance 1 .

Since $W=W^{\mathrm{T}}$ and $W^{\mathrm{T}}=W^{\mathrm{T}}$ we shall only work with $W$ and $W^{\mathrm{T}}$. For this reason we shall use slightly different notation that in the previous sections. For $\epsilon \in \mathbb{Z}_{2}=$ $\{-1,1\}$, we let

$$
W^{(\epsilon)}= \begin{cases}W & \epsilon=1, \\ W^{\Gamma} & \epsilon=-1 .\end{cases}
$$


Thus, our goal will be to consider, $W^{\left(\epsilon_{1}\right)} \ldots W^{\left(\epsilon_{n}\right)}$, an arbitrary word in $W$ and $W^{\mathrm{T}}$ and find its limiting expectation.

Theorem 8.1 With the notations from above, we have that

$$
\mathrm{E}\left(\operatorname{tr} \otimes \operatorname{tr}\left(W^{\left(\epsilon_{1}\right)} \cdots W^{\left(\epsilon_{n}\right)}\right)\right)=\sum_{\pi \in \mathcal{P}_{2}( \pm n)}\left(\frac{p}{d_{1} d_{2}}\right)^{\#(\pi \delta) / 2} d_{1}^{g(\pi)} d_{2}^{g(\epsilon \pi \epsilon)},
$$

where

$$
g(\pi)=\#\left(\gamma \delta \gamma^{-1} \vee \pi\right)+\#(\pi \delta) / 2-(n+1)
$$

and $\epsilon \in S( \pm n)$ is, as before, given by

$$
\epsilon(k)= \begin{cases}k, & \text { if } \epsilon_{|k|}=1, \\ -k & \text { if } \epsilon_{|k|}=-1 .\end{cases}
$$

Proof We have

$$
\begin{aligned}
& \operatorname{Tr} \otimes \operatorname{Tr}\left(W^{\left(\epsilon_{1}\right)} \ldots W^{\left(\epsilon_{n}\right)}\right) \\
& =\sum_{\substack{i_{ \pm 1}, \ldots, i_{ \pm n}=1 \\
i=i \circ \gamma \delta \gamma^{-1}}}^{d_{1}} \operatorname{Tr}\left(W\left(i_{1}, i_{-1}\right)^{\left(\epsilon_{1}\right)} \ldots W\left(i_{n} i_{-n}\right)^{\left(\epsilon_{n}\right)}\right) \\
& =\sum_{i_{ \pm 1}, \ldots, i_{ \pm n}} \sum_{\substack{ \pm 1 \\
k=k \circ \gamma \delta \gamma^{-1}}}^{d_{2}}\left(W\left(i_{1}, i_{-1}\right)^{\left(\epsilon_{1}\right)}\right)_{k_{1} k_{-1}} \cdots\left(W\left(i_{n} i_{-n}\right)^{\left(\epsilon_{n}\right)}\right)_{k_{n} k_{-n}} \\
& =\sum_{\substack{i_{ \pm 1}, \ldots, i_{ \pm n} \\
j_{j 1}, \ldots, j_{+n}=1 \\
j=j \circ \epsilon \delta \gamma^{-1} \epsilon}}^{d_{2}} W\left(i_{1}, i_{-1}\right)_{j_{1} j_{-1}} \cdots W\left(i_{n} i_{-n}\right)_{j_{n} j_{-n}} \\
& =\left(d_{1} d_{2}\right)^{-n} \sum_{\substack{i_{ \pm 1}, \ldots, i_{ \pm n} \\
j_{ \pm 1}, \ldots, j_{ \pm n}}} \sum_{\substack{1 \\
1}, \ldots, k_{n}=1}^{p} g_{j_{1} k_{1}}^{\left(i_{1}\right)} g_{j_{-1} k_{1}}^{\left(i_{-1}\right)} \cdots g_{j_{n} k_{n}}^{\left(i_{n}\right)} g_{j_{-n} k_{n}}^{\left(i_{-n}\right)} .
\end{aligned}
$$

In line 3 we momentarily break with our previous convention about $W^{(e)}$ indicating whether or not we take a partial transpose; in this case $W\left(i_{u}, i_{-u}\right)^{(-1)}$ means take the transpose of the $d_{2} \times d_{2}$ matrix $W\left(i_{u}, i_{-u}\right)$. In passing from line 3 to line 4 above, we let $j=k \circ \epsilon$.

Now

$$
\mathrm{E}\left(g_{j_{1} k_{1}}^{\left(i_{1}\right)} g_{j_{-1} k_{1}}^{\left(i_{-1}\right)} \cdots g_{j_{n} k_{n}}^{\left(i_{n}\right)} g_{j_{-n} k_{n}}^{\left(i_{-n}\right)}\right)=\sum_{\pi \in \mathcal{P}_{2}( \pm n)} \prod_{(r, s) \in \pi} \mathrm{E}\left(g_{j_{r} k_{r}}^{\left(i_{r}\right)} g_{j_{s} k_{s}}^{\left(i_{s}\right)}\right) .
$$

On the right-hand side in the expression above we are extending $k$ as a function from $[n]$ to $[p]$ to a function on $[ \pm n]$ by requiring $k_{r}=k_{-r}$. This means $k=k \circ \delta$. Now $\mathrm{E}\left(g_{j_{r} k_{r}}^{\left(i_{r}\right)} g_{j_{s} k_{s}}^{\left(i_{s}\right)}\right)=0$ unless $i_{r}=i_{s}, j_{r}=j_{s}$, and $k_{r}=k_{s}$, in which case it is 1 . Thus,

$$
\mathrm{E}\left(\operatorname{Tr} \otimes \operatorname{Tr}\left(W^{\left(\epsilon_{1}\right)} \cdots W^{\left(\epsilon_{n}\right)}\right)\right)=\sum_{\pi \in \mathcal{P}_{2}( \pm n)} d_{1}^{\#\left(\gamma \delta \gamma^{-1} \vee \pi\right)-n} d_{2}^{\#\left(\epsilon \gamma \delta \gamma^{-1} \epsilon \vee \pi\right)-n} p^{\#(\pi \delta) / 2} .
$$


Since we require $k=k \circ \pi$ and $k=k \circ \delta$ we must have $k=k \circ \pi \delta$. Now as noted in Lemma 4.1 the cycles of $\pi \delta$ appear in pairs where one part of a pair is the conjugate by $\delta$ of the other. Since $k$ is a function on $[n], \#(\pi \delta)$ double counts the degrees of freedom. Hence the exponent of $p$ is $\#(\pi \delta) / 2$. Thus,

$$
\begin{aligned}
\mathrm{E}(\operatorname{tr} \otimes & \left.\operatorname{tr}\left(W^{\left(\epsilon_{1}\right)} \cdots W^{\left(\epsilon_{n}\right)}\right)\right) \\
= & \sum_{\pi \in \mathcal{P}_{2}( \pm n)}\left(\frac{p}{d_{1} d_{2}}\right)^{\#(\pi \delta) / 2} d_{1}^{\#\left(\gamma \delta \gamma^{-1} \vee \pi\right)+\#(\pi \delta) / 2-(n+1)} \\
& \times d_{2}^{\#\left(\epsilon \gamma \delta \gamma^{-1} \epsilon \vee \pi\right)+\#(\pi \delta) / 2-(n+1)} .
\end{aligned}
$$

Finally, note that

$$
\begin{aligned}
\#\left(\epsilon \gamma \delta \gamma^{-1} \epsilon \vee \pi\right)+\#(\pi \delta) / 2-(n+1) & =\frac{1}{2} \#\left(\gamma \delta \gamma^{-1} \epsilon \pi \epsilon\right)+\#(\epsilon \pi \delta \epsilon) / 2-(n+1) \\
& =g(\epsilon \pi \epsilon),
\end{aligned}
$$

hence the conclusion.

Next we shall show that $g(\pi) \leq 0$ and $g(\epsilon \pi \epsilon) \leq 0$ for all $\epsilon$ and $\pi$, and find for which pairings $\pi$ we have equality.

Lemma 8.2 Let $\pi \in \mathcal{P}_{2}( \pm n)$ be a pairing such that there is $(r, s) \in \pi$ with the same sign. Then $g(\pi)<0$.

Proof Since $\pi$ connects two elements with the same sign, $\pi \delta$ connects two elements with opposite signs. Then the subgroup generated by $\gamma \delta \gamma^{-1} \delta$ and $\pi \delta$ acts transitively on $[ \pm n]$. Thus,

$$
\#(\pi \delta)+\#\left((\pi \delta)^{-1} \gamma \delta \gamma^{-1} \delta\right)+\#\left(\gamma \delta \gamma^{-1} \delta\right) \leq 2(n+1)
$$

We have

$$
2 \#\left(\gamma \delta \gamma^{-1} \vee \pi\right)=\#\left(\gamma \delta \gamma^{-1} \pi\right)=\#\left(\gamma \delta \gamma^{-1} \delta \delta \pi\right)=\#\left((\pi \delta)^{-1} \gamma \delta \gamma^{-1} \delta\right) .
$$

Thus, $g(\pi)=\#\left(\gamma \delta \gamma^{-1} \vee \pi\right)+\#(\pi \delta) / 2-(n+1) \leq-1$.

Lemma 8.3 Suppose $\pi \in \mathcal{P}_{2}( \pm n)$ and $\pi$ only connects elements of opposite sign. Then $\pi \delta$ leaves $[n]$ invariant and $g(\pi) \leq 0$ with equality only if $\left.\pi \delta\right|_{[n]}$ is a non-crossing permutation.

Proof Since both $\pi$ and $\delta$ switch signs, $\pi \delta$ preserves signs. Thus, $\pi \delta$ leaves $[n]$ invariant. By Lemma 4.1 we have $\#(\pi \delta)=2 \#\left(\left.\pi \delta\right|_{[n]}\right)$. Also,

$$
2 \#\left(\gamma \delta \gamma^{-1} \vee \pi\right)=\#\left(\gamma \delta \gamma^{-1} \delta \delta \pi\right)=\#\left((\pi \delta)^{-1} \gamma \delta \gamma^{-1} \delta\right)=2 \#\left(\left(\left.\pi \delta\right|_{[n]}\right)^{-1} \gamma\right) \text {. }
$$

Hence,

$$
g(\pi)=\#\left(\gamma \delta \gamma^{-1} \vee \pi\right)+\#(\pi \delta) / 2-(n+1) \leq 0
$$

with equality only if $\left.\pi \delta\right|_{[n]}$ is a non-crossing permutation.

Lemma 8.4 Let $\epsilon \in \mathbb{Z}_{2}^{n}$ and $\pi \in \mathcal{P}_{2}( \pm n)$. Then $g(\epsilon \pi \epsilon)<0$ unless $\epsilon \pi \delta \epsilon$ leaves $[n]$ invariant. If $\epsilon \pi \delta \epsilon$ leaves $[n]$ invariant, then $g(\epsilon \pi \epsilon) \leq 0$ with equality only if $\left.\epsilon \pi \delta \epsilon\right|_{[n]}$ is a non-crossing permutation. 
Proof By Lemma 8.3 we have $g(\epsilon \pi \epsilon)<0$ unless $\epsilon \pi \epsilon \delta=\epsilon \pi \delta \epsilon$ leaves [ $n$ ] invariant. If $\epsilon \pi \delta \epsilon$ leaves $[n]$ invariant, then again by Lemma 8.3, we have $g(\epsilon \pi \epsilon) \leq 0$ with equality only if $\left.\epsilon \pi \delta \epsilon\right|_{[n]}$ is a non-crossing permutation.

Lemma 8.5 Let $\epsilon \in \mathbb{Z}_{2}^{n}$ and $\pi \in \mathcal{P}_{2}( \pm n)$. Suppose $\pi \delta$ leaves $[n]$ invariant. Then $\epsilon \pi \delta \epsilon$ leaves $[n]$ invariant if and only if $\epsilon$ is constant on the cycles of $\pi \delta$.

Proof Suppose $\left(i_{1}, \ldots, i_{k}\right)$ is a cycle of $\pi \delta$. All these elements must have the same sign. The corresponding cycle of $\epsilon \pi \delta \epsilon$ is $\left(\epsilon\left(i_{1}\right), \ldots, \epsilon\left(i_{k}\right)\right)$. The elements of $\epsilon \pi \delta \epsilon$ is $\left(\epsilon\left(i_{1}\right), \ldots, \epsilon\left(i_{k}\right)\right)$ have the same sign if and only if $\epsilon$ is constant on $\epsilon \pi \delta \epsilon$ is $\left(\epsilon\left(i_{1}\right), \ldots, \epsilon\left(i_{k}\right)\right)$.

The following theorem is the main result of this section. Recall from Lemma 4.4 that if $\epsilon$ is constant on the cycles of $\sigma$, then we obtain $\sigma_{\epsilon}$ from $\sigma$ by reversing the cycles on which $\epsilon=-1$.

\section{Theorem 8.6 We have}

$$
\lim _{d_{1}, d_{2} \rightarrow \infty} \mathrm{E}\left(\operatorname{tr} \otimes \operatorname{tr}\left(W^{\left(\epsilon_{1}\right)} \ldots W^{\left(\epsilon_{n}\right)}\right)\right)=\sum_{\sigma \in S_{N C}(n)} c^{\#(\sigma)},
$$

where the sum runs over all non-crossing permutations $\sigma$ such that $\epsilon$ is constant on the cycles of $\sigma$ and $\sigma_{\epsilon}$ is also non-crossing.

Proof In the formula from Theorem 8.1, only the pairings $\pi$ such that $g(\pi)=$ $g(\epsilon \pi \epsilon)=0$ will contribute to the summation when $d_{1}, d_{2} \rightarrow \infty$.

Recall that $\mathcal{P}_{2}^{\delta}( \pm n)$ denotes the pairings $\pi$ of $[ \pm n]$ such that $\pi \delta$ leaves $[n]$ invariant. For such a $\pi$ we let $\sigma=\left.\pi \delta\right|_{[n]}$ be the corresponding permutation. We already noted that this is a bijection from $\mathcal{P}_{2}^{\delta}( \pm n)$ to $S_{n}$ and $\pi \delta=\delta \sigma^{-1} \delta \sigma$. From Lemma 8.3, the condition $g(\pi)=0$ implies that $\sigma=\delta \sigma^{-1} \delta \sigma_{[n]}$ is noncrossing.

According to Lemmas 8.4 and 8.5, the condition $g(\epsilon \pi \epsilon)=0$ implies that $\epsilon$ is constant on the cycles of $\sigma$. As in Lemma $4.4, \epsilon \pi \delta \epsilon=\delta \sigma_{\epsilon}^{-1} \delta \sigma_{\epsilon}$. Therefore,

$$
\begin{aligned}
\#\left(\epsilon \gamma \delta \gamma^{-1} \epsilon \vee \pi\right) & =\frac{1}{2} \#\left(\gamma \delta \gamma^{-1} \delta(\epsilon \pi \delta \epsilon)^{-1}\right) \\
& =\frac{1}{2} \#\left(\left(\delta \sigma_{\epsilon}^{-1} \delta \sigma_{\epsilon}\right)^{-1} \gamma \delta \gamma^{-1} \delta\right)=\#\left(\sigma_{\epsilon}^{-1} \gamma\right),
\end{aligned}
$$

which gives

$$
g(\epsilon \pi \epsilon)=\#\left(\sigma_{\epsilon}\right)+\#\left(\sigma_{\epsilon}^{-1} \gamma\right)-(n+1)
$$

hence the formula (4.1) gives that $g(\epsilon \pi \epsilon) \leq 0$ with equality if and only if $\sigma_{\epsilon}$ is noncrossing.

An immediate consequence of Theorem 8.6 is part (i) of the following result. 
Theorem 8.7 Suppose that $p /\left(d_{1} d_{2}\right) \rightarrow c$.

(i) If $d_{1}, d_{2} \rightarrow \infty$, then $W^{\Gamma}$ is asymptotically a shifted semi-circular operator with $\kappa_{1}=\kappa_{2}=c$.

(ii) If $d_{1} \rightarrow \infty$ and $d_{2} \geq 2$ is fixed, then the asymptotic distribution of $d_{2} W^{\Gamma}$, equals the distribution of the difference of two free variables with Marchenko-Pastur laws, the first of parameter $c d_{2} \frac{d_{2}+1}{2}$ and the second of parameter $c d_{2} \frac{d_{2}-1}{2}$.

(iii) If $d_{1}$ is fixed and $d_{2} \rightarrow \infty$, then the asymptotic distribution of $d_{1} W^{\Gamma}$, equals the distribution of the difference of two free variables with Marchenko-Pastur laws, the first of parameter $c d_{1} \frac{d_{1}+1}{2}$ and the second of parameter $c d_{1} \frac{d_{1}-1}{2}$.

Proof Letting $\epsilon_{j}=-1$ for all $j=1, \ldots, n$ in Theorem 8.1, we obtain that

$$
E \circ \operatorname{tr} \otimes \operatorname{tr}\left(\left(W^{\Gamma}\right)^{n}\right)=\sum_{\pi \in \mathcal{P}_{2}( \pm n)}\left(\frac{p}{d_{1} d_{2}}\right)^{\#(\pi \delta) / 2} d_{1}^{g(\pi)} d_{2}^{g(\delta \pi \delta)} .
$$

Suppose first that $d_{1}, d_{2} \rightarrow \infty$. Then, in the summation from (8.1), only terms with $g(\pi)=g(\delta \pi \delta)=0$ will contribute to the limit. From Theorem 8.6, this is equivalent to both $\sigma$ and $\sigma_{\delta}$ be noncrossing. But $\sigma_{\delta}=\sigma^{-1}$ so Lemma 5.1 implies that $\sigma$ has only cycles of length 1 or 2 , hence part (i) is proved.

Suppose now that $d_{1} \rightarrow \infty$ and $d_{2}$ is fixed. Then only $\pi$ such that $g(\pi)=0$ will contribute to the limit in the summation (8.1). Applying Lemma 8.3 again, this is equivalent to $\pi=\sigma \delta \sigma^{-1}$, for $\sigma$ a non-crossing permutation on $[n]$. In this case, we have that $g(\delta \pi \delta)=g\left(\delta \sigma \delta \sigma^{-1} \delta\right)$.

Also, $\#\left(\gamma \delta \gamma^{-1} \vee \delta \pi \delta\right)=\frac{1}{2} \#\left(\gamma \delta \gamma^{-1} \delta \sigma \delta \sigma^{-1} \delta\right)$, and, if $k \in[n]$, we have that

$$
\begin{aligned}
\gamma \delta \gamma^{-1} \delta \sigma \delta \sigma^{-1} \delta(k) & =\gamma \delta \gamma^{-1} \delta \sigma(k)=\gamma \sigma(k), \\
\gamma \delta \gamma^{-1} \delta \sigma \delta \sigma^{-1} \delta(-k) & =\gamma \delta \gamma^{-1} \delta \sigma \delta \sigma^{-1}(k)=\gamma \delta \gamma^{-1} \delta \sigma\left(-\sigma^{-1}(k)\right) \\
& =\gamma \delta \gamma^{-1}\left(\sigma^{-1}(k)\right)=\gamma^{-1}\left(\sigma^{-1}(k)\right)=(\sigma \circ \gamma)^{-1}(k) .
\end{aligned}
$$

Moreover,

$$
\#((\delta \pi \delta) \delta)=\#(\delta \pi)=\#\left((\pi \delta)^{-1}\right)=2 \#(\sigma),
$$

hence, Lemma 5.5 gives that

$$
g(\delta \pi \delta)=\#(\gamma \sigma)+\#(\sigma)-(n+1)=\#(\sigma)+e(\sigma)-n
$$

so equation (8.1) becomes

$$
\lim _{d_{1} \rightarrow \infty} E \circ \operatorname{tr} \otimes \operatorname{tr}\left(\left(W^{\Gamma}\right)^{n}\right)=\sum_{\sigma \in S_{N C}(n)} c^{\#(\sigma)} d_{2}^{e(\sigma)+\#(\sigma)-n} .
$$

Thus,

$$
\lim _{d_{1} \rightarrow \infty} E \circ \operatorname{tr} \otimes \operatorname{tr}\left(\left(d_{2} W^{\Gamma}\right)^{n}\right)=\sum_{\sigma \in S_{N C}(n)}\left(c d_{2}\right)^{\#(\sigma)} d_{2}^{e(\sigma)}=\sum_{\sigma \in S_{N C}(n)} \kappa_{\sigma},
$$

where $\kappa_{n}=c d_{2}$ for $n$ odd and $\kappa_{n}=c d_{2}^{2}$ for $n$ even. The conclusion follows, because $\kappa_{n}=\left(c d_{2}\right) \frac{d_{2}+1}{2}+(-1)^{n}\left(c d_{2}\right) \frac{d_{2}-1}{2}$ (see the proof of Theorem 5.6). The case $d_{1}$ fixed and $d_{2} \rightarrow \infty$ is similar. 
Theorem 8.8 If both $d_{1}, d_{2} \rightarrow \infty$, then $\left\{W, W^{\mathrm{\Gamma}}\right\}$ is an asymptotically free family.

Proof The result is a consequence of Theorems 8.6 and 8.7.

Remark 8.9 For $W$ a real Wishart random matrix, $W^{\Gamma}$ is not asymptotically free from $W$ if $d_{1}$ is fixed or if $d_{2}$ is fixed.

Indeed, for $n=2$ and $\epsilon_{1}=1$ and $\epsilon_{2}=-1$, the formula from Theorem 8.1 gives

$$
E \circ \operatorname{tr} \otimes \operatorname{tr}\left(W W^{\Gamma}\right)=\sum_{\pi \in \mathcal{P}_{2}( \pm 2)}\left(\frac{p}{d_{1} d_{2}}\right)^{\#(\pi \delta) / 2} d_{1}^{g(\pi)} d_{2}^{g(\epsilon \pi \epsilon)} .
$$

There are only 3 pairings in $P_{2}( \pm 2): \pi_{1}=(1,-1),(2,-2), \pi_{2}=(1,2),(-1,-2)$, and $\pi_{3}=(1,-2),(-1,2)$. Direct calculations give that $\pi_{1} \delta=\mathrm{id}, \pi_{2} \delta=(1,-2),(-1,2)$ and $\pi_{2} \delta=(1,2),(-1,-2)$.

Moreover, $\epsilon \pi_{1} \epsilon=\pi_{1}$, while $\epsilon \pi_{2} \epsilon=\pi_{3}$ and $\epsilon \pi_{3} \epsilon=\pi_{2}$; also, for $n=2$, we have that $\gamma \delta \gamma^{-1}=(1,-2),(-1,2)$. Therefore, $g\left(\pi_{1}\right)=g\left(\pi_{2}\right)=0$ and $g\left(\pi_{3}\right)=1$, so

$$
E \circ \operatorname{tr} \otimes \operatorname{tr}\left(W W^{\mathrm{\Gamma}}\right)=\left(\frac{p}{d_{1} d_{2}}\right)^{2}+\left(\frac{p}{d_{1} d_{2}}\right) \cdot\left(\frac{1}{d_{1}}+\frac{1}{d_{2}}\right),
$$

and the second term in the equation above does not cancel asymptotically unless both $d_{1}, d_{2} \rightarrow \infty$.

\section{References}

[1] O. Arizmendi, I. Nechita, and C. Vargas, On the asymptotic distribution of block-modified random matrices. J. Math. Phys. 57(2016), no. 1, 015216. http://dx.doi.org/10.1063/1.4936925

[2] G. Aubrun, Partial transposition of random states and non-centered semicircular distributions. Random Matrices Theory Appl. 1(2012), no. 2, 1250001. http://dx.doi.org/10.1142/S2010326312500013

[3] T. Banica and I. Nechita, Asymptotic eigenvalue distributions of block-transposed Wishart matrices. J. Theor. Probab. 26(2013), 855-869. http://dx.doi.org/10.1007/s10959-012-0409-4

[4] P. Biane, Some properties of crossings and partitions. Discrete Mathematics 175(1997), 41-53. http://dx.doi.org/10.1016/S0012-365X(96)00139-2

[5] R. Cori, Un code pour les graphes planaires et ses applications. Astérisque, 27, Société Mathématique de France, Paris, 1975.

[6] M. Fukuda and P. Śniady, Partial transpose of random quantum states: exact formulas and meanders. J. Math. Phys. 54(2013), no. 4, 042202. http://dx.doi.org/10.1063/1.4799440

[7] S. Janson, Gaussian Hilbert spaces. Cambridge Tracts in Mathematics, 129, Cambridge University Press, Cambridge, 1997.

[8] J. A. Mingo and M. Popa, Real second order freeness and Haar orthogonal matrices. J. Math. Phys. 54(2013), no. 5, 051701. http://dx.doi.org/10.1063/1.4804168

[9] J. A. Mingo and M. Popa, Freeness and the transposes of unitarily invariant random matrices. J. Funct. Anal. 271(2016), 883-921. http://dx.doi.org/10.1016/j.jfa.2016.05.006

[10] J. A. Mingo and R. Speicher, Free probability and random matrices. Fields Institute Monographs, 35, Springer, New York; Fields Institute for Research in Mathematical Sciences, Toronto, ON, 2017.

[11] A. Nica and R. Speicher, Lectures on the combinatorics of free probability. Cambridge University Press, Cambridge, 2006.

[12] C. E. I. Redelmeier, Genus expansion for real Wishart matrices. J. Theoret. Probab. 24(2011), 1044-1062. http://dx.doi.org/10.1007/s10959-010-0278-7

[13] C. E. I. Redelmeier, Real second-order freeness and the asymptotic real second-order freeness of several real matrix models. Int. Math. Res. Not. IMRN 2014, no. 12, 3353-3395. 
Department of Mathematics and Statistics, Queen's University, Jeffery Hall, Kingston, Ontario K7L 3N6 e-mail: mingo@mast.queensu.ca

Department of Mathematics, The University of Texas at San Antonio, One UTSA Circle, San Antonio, TX 78249, USA

and

Institute of Mathematics "Simion Stoilow" of the Romanian Academy, P.O. Box 1-764, Bucharest, RO70700, Romania

e-mail: mihai.popa@utsa.edu 